MATHEMATICS OF COMPUTATION

Volume 77, Number 261, January 2008, Pages 421-446

S 0025-5718(07)01839-X

Article electronically published on September 12, 2007

\title{
STATISTICAL PROPERTIES OF GENERALIZED DISCREPANCIES
}

\author{
CHRISTINE CHOIRAT AND RAFFAELLO SERI
}

\begin{abstract}
When testing that a sample of $n$ points in the unit hypercube $[0,1]^{d}$ comes from a uniform distribution, the Kolmogorov-Smirnov and the Cramér-von Mises statistics are simple and well-known procedures. To encompass these measures of uniformity, Hickernell introduced the so-called generalized $\mathcal{L}^{p}$-discrepancies. These discrepancies can be used in numerical integration through Monte Carlo and quasi-Monte Carlo methods, design of experiments, uniformity testing and goodness-of-fit tests. The aim of this paper is to derive the statistical asymptotic properties of these statistics under Monte Carlo sampling. In particular, we show that, under the hypothesis of uniformity of the sample of points, the asymptotic distribution is a complex stochastic integral with respect to a pinned Brownian sheet. On the other hand, if the points are not uniformly distributed, then the asymptotic distribution is Gaussian.
\end{abstract}

\section{INTRODUCTION}

The Koksma-Hlawka inequality (see [Nie92, p. 18) is a well-known bound on the error of Monte Carlo and quasi-Monte Carlo integration: it majorizes the error through the variation of the integrand in the sense of Hardy and Krause times the star discrepancy of the random or quasi-random points (Nie92, Definition 2.1). The star discrepancy has a remarkable statistical interpretation since, when the integrand is defined on the real hypercube $[0,1]^{d}$, it has the same form as the Kolmogorov-Smirnov statistic to test uniformity.

In a series of important papers, Hickernell ([Hic96 and Hic97, Hic98a, Hic98b]) has introduced the generalized $\mathcal{L}^{p}$-discrepancies $D_{p}\left(\mathcal{P}_{n}\right)$ based on the sample of $n$ points $\mathcal{P}_{n}[1$ they extend the star discrepancy and allow for measuring the degree of nonuniformity of the sample and the efficiency of the numerical integration procedure. The links of these figures of merit with goodness-of-fit statistics and with optimal design of experiments have been pointed out by Hickernell ([Hic99]) and Fang et al. ([FMW02]).

Liang et al. ([LFHL01]) have started an investigation of the asymptotic properties of $D_{p}\left(\mathcal{P}_{n}\right)$ in the case $p=2$ : they show that, under the hypothesis of uniformity,

Received by the editor October 22, 2004 and, in revised form, May 11, 2005.

2000 Mathematics Subject Classification. Primary 65D30, 60F05, 68U20, 65C05, 11K45.

Key words and phrases. Generalized discrepancies, testing uniformity, Monte Carlo, quasiMonte Carlo, limit distribution.

We thank Peter Hellekalek, Søren Johansen, Peter E. Jupp for useful comments on a previous version of this paper and Kendall E. Atkinson and David E. Edmunds for useful references. We also thank an anonymous referee for comments and suggestions that led to improve the paper.

${ }^{1}$ In $D_{p}\left(\mathcal{P}_{n}\right)$ we make explicit the dependence on the indices $p$ and $n$.

(C)2007 American Mathematical Society 421

Reverts to public domain 28 years from publication 
$D_{2}\left(\mathcal{P}_{n}\right)$ converges almost surely to 0 , but they do not derive its asymptotic distribution. Then, they obtain two alternative statistics, strictly linked to this one, which can be used to statistically test the efficiency of numerical integration procedures 2 The aim of this paper is to complete the analysis of [LFHL01] and to develop some further properties of the test statistics proposed by Hickernell (Hic98a, Hic98b]). The interest of these results is more theoretical than practical: indeed, the asymptotic distributions that we obtain are quite difficult to compute (except in the case $p=2$; see [CS05b]), but these results yield precise information about the rate of convergence of these statistics to 0 when the sample $\mathcal{P}_{n}$ is uniformly scattered and the rate of divergence when the sample is not uniformly scattered.

As concerns the generalized discrepancy $D_{p}\left(\mathcal{P}_{n}\right)$, we show that $D_{p}\left(\mathcal{P}_{n}\right)$ converges almost surely to 0 if and only if the sample $\mathcal{P}_{n}$ is uniformly scattered (as defined below) on the unit hypercube $[0,1]^{d}$. Under this distributional hypothesis, we obtain a rate of convergence towards zero: first of all, we show that $D_{p}\left(\mathcal{P}_{n}\right)$ is $O_{\mathbb{P}}\left(n^{-1 / 2}\right) 3$ that is, $\sqrt{n} \cdot D_{p}\left(\mathcal{P}_{n}\right)$ converges in distribution to a nontrivial random variable whose form is provided in terms of a stochastic integral; moreover, we prove that a Law of the Iterated Logarithm holds for the generalized discrepancy.

We show that, in the case $p=2$, the asymptotic distribution $\left[D_{2}\left(\mathcal{P}_{n}\right)\right]^{2}$ can be written as a weighted infinite mixture of $\chi^{2}$ distributions and is a particular instance of what is called Gaussian Chaos. While the asymptotic distribution for $p \neq 2$ is difficult to obtain, in this special case it can be computed using the algorithm presented in CS05a. However, it can be shown that this result holds for a much more general class of discrepancies (the generalized $\mathcal{L}^{2}$-discrepancies, the classical and the dyadic diaphonies, the weighted spectral test, the serial and the overlapping serial test, etc.). This is the object of a companion paper (CS05b]).

Moreover, we derive the asymptotic distribution of $D_{p}\left(\mathcal{P}_{n}\right)$ under the hypothesis that the sample $\mathcal{P}_{n}$ does not come from a uniform distribution. In this case, for finite values of $p$, the asymptotic distribution is Gaussian, but an alternative representation can be given as a stochastic integral. A limited simulation study, in Section 4 . confirms these theoretical findings for the case of generalized $\mathcal{L}^{2}$-discrepancies.

The results shown in this paper can be generalized along several lines. A particularly interesting topic would be the extension to sequences of points that are not independent and identically distributed, but of particular relevance to Numerical Analysis, such as scrambled digital nets (see [HHW03 for simulation results). This will be left to future work.

Since the results make large use of statistical and probabilistic tools, we need to introduce some notation. In what follows, the term uniformly scattered is used in the sense of Fang and Wang (FW94, p. 18, see also Remark 1.3) to indicate that the points are uniformly distributed in the sense of Niederreiter (Nie92], p. 13) (we use the new definition in order to avoid confusion with the related statistical concept). We introduce a notation that is typical of empirical process theory: for

${ }^{2}$ Other authors have recently investigated similar discrepancies (Leeb in Lee96a Lee96b, Lee02], Hoogland and Kleiss in [HK96a, HK96b, HK97], James et al. in [JHK97], Hoogland et al. in [HJK98, and van Hameren et al. in vHKH97]).

${ }^{3}$ We say that $X_{n}$ is $O_{\mathbb{P}}\left(r_{n}\right)$ if, for each $\varepsilon>0$, there exists $M>0$ such that

$$
\mathbb{P}\left\{\frac{\left|X_{n}\right|}{r_{n}}>M\right\}<\varepsilon \quad \forall n,
$$

where $M$ and $\varepsilon$ do not depend on $n$. 
a probability space $(\mathcal{X}, \mathcal{B}, \mathbb{P})$ and a measurable function $f: \mathcal{X} \rightarrow \mathbb{R}^{k}$, we write $\mathbb{P} f=\int_{\mathcal{X}} f \mathrm{~d} \mathbb{P}$ to indicate the integral of $f$ with respect to $\mathbb{P}$. If there is any doubt, we write the variable of integration in capital letters $\left(\mathbb{P} f(X)=\int_{\mathcal{X}} f(x) \mathbb{P}(\mathrm{d} x)\right)$. Unless otherwise stated, we will assume that $\mathbb{P}$ is the Lebesgue measure on the unit hypercube $[0,1]^{d}$, also written as $\lambda^{\otimes d}$ 贯 In the following, for a $n$-sample $\mathcal{P}_{n}$, we define the empirical measures $\lambda_{n}^{\otimes d}$ and the associated integrals a:5

$$
\begin{gathered}
\lambda_{n}^{\otimes d}=\frac{1}{n} \sum_{\mathbf{z} \in \mathcal{P}_{n}} \delta_{\mathbf{z}}, \\
\lambda_{n}^{\otimes d} f=\int f \mathrm{~d} \lambda_{n}^{\otimes d}=\frac{1}{n} \sum_{\mathbf{z} \in \mathcal{P}_{n}} f(\mathbf{z}),
\end{gathered}
$$

where $\delta_{\mathbf{z}}$ is the Dirac measure concentrated in $\mathbf{z}$. Moreover, we define as $|A|$ the number of points in the set $A$ and as $\operatorname{Vol}([\mathbf{0}, \mathbf{x}))$ the volume of the rectangular solid $[\mathbf{0}, \mathbf{x})$. For any index set $u \subseteq\{1, \ldots, d\}$, we denote by $|u|$ its cardinality, by $[0,1]^{u}$ the $|u|$-dimensional unit hypercube and by $\mathbf{x}_{u}$ a $|u|$-dimensional vector containing the elements of $\mathbf{x}$ indexed by the elements of $u$.

\section{AsYMPTOTIC RESULTS FOR $\mathcal{L}^{p}$-DISCREPANCIES UNDER UNIFORMITY}

The generalized discrepancies $D_{p}\left(\mathcal{P}_{n}\right)$ have been introduced in Hickernell ([Hic96, Hic98a, Hic98b]) as a generalization of some figures of merit that have arisen in the literature. In the case $1 \leq p \leq+\infty$ (see equation (3.8b) in [Hic98a ) they are defined by the equation

$$
\begin{aligned}
D_{p}\left(\mathcal{P}_{n}\right)= & {\left[\sum_{u \neq \varnothing}\left\|\beta^{|u|} \cdot D_{p, u}\left(\mathcal{P}_{n, u}\right)\right\|_{\mathcal{L}^{p}}^{p}\right]^{1 / p} } \\
= & {\left[\sum_{u \neq \varnothing} \int_{[0,1]^{u}} \mid \beta^{|u|} \cdot\left\{\prod_{j \in u} \mu^{\prime}\left(x_{j}\right)\right.\right.} \\
& \left.\left.\quad-\frac{1}{n} \sum_{\mathbf{z} \in \mathcal{P}_{n}} \prod_{j \in u}\left[\mu^{\prime}\left(x_{j}\right)+x_{j}-1_{\left\{x_{j}>z_{j}\right\}}\right]\right\}\left.\right|^{p} \mathrm{~d} \mathbf{x}_{u}\right]^{1 / p},
\end{aligned}
$$

where $u$ is a subset of the set $\{1, \ldots, d\}, \mathcal{P}_{n, u}$ denotes the projection of the sample $\mathcal{P}_{n}$ on the unit cube $[0,1]^{u}, \beta$ is an arbitrary given positive constant and $\mu(\cdot)$ is an arbitrary function satisfying

$$
\mu \in\left\{f: \frac{\mathrm{d} f}{\mathrm{~d} x} \in \mathcal{L}^{\infty}([0,1]) \text { and } \int_{0}^{1} f(x) \mathrm{d} x=0\right\} .
$$

\footnotetext{
${ }^{4}$ The $\otimes$ sign is used to remind us that it is the $d$-dimensional product Lebesgue measure. Strictly speaking, the uniform distribution should be written as $\left.\lambda^{\otimes d}\right|_{[0,1]}$, the $d$-dimensional Lebesgue measure restricted to $[0,1]^{d}$, but with a small abuse of notation we will prefer to write $\lambda^{\otimes d}$.

${ }^{5}$ The subscript $n$ reminds us that the measure is the empirical counterpart of $\lambda^{\otimes d}$.
} 
We remark that in the case $p=+\infty$, the previous formula becomes

$$
\begin{aligned}
D_{\infty}\left(\mathcal{P}_{n}\right) & =\max _{u}\left\|\beta^{|u|} \cdot D_{\infty, u}\left(\mathcal{P}_{n, u}\right)\right\|_{\mathcal{L}^{\infty}} \\
& =\max _{u} \underset{\mathbf{x}_{u} \in[0,1]^{u}}{\operatorname{ess} \beta^{u u \mid}}\left|\prod_{j \in u} \mu^{\prime}\left(x_{j}\right)-\frac{1}{n} \sum_{\mathbf{z} \in \mathcal{P}_{n}} \prod_{j \in u}\left[\mu^{\prime}\left(x_{j}\right)+x_{j}-1_{\left\{x_{j}>z_{j}\right\}}\right]\right| .
\end{aligned}
$$

Most of our results can be easily extended to weighted $\mathcal{L}^{p}$-discrepancies in the spirit of [SW98] and [LP03], replacing $\beta^{|u|}$ with $\prod_{j \in u} \gamma_{j}$, and in the spirit of [SWW04, substituting $\beta^{|u|}$ with $\gamma_{d,|u|}$ (refer to these papers for definitions) 6 When the sample $\mathcal{P}_{n}$ is uniformly scattered, it is crucial to remark that a recurrent element of the previous formulas can be written as the deviation of the empirical distribution from the uniform one:

$$
\begin{aligned}
\prod_{j \in u} \mu^{\prime}\left(x_{j}\right)-\frac{1}{n} \sum_{\mathbf{z} \in \mathcal{P}_{n}} \prod_{j \in u}\left[\mu^{\prime}\left(x_{j}\right)+x_{j}-1_{\left\{x_{j}>z_{j}\right\}}\right] \\
=\lambda^{\otimes d}\left\{\prod_{j \in u}\left[\mu^{\prime}\left(x_{j}\right)+x_{j}-1_{\left\{x_{j}>Z_{j}\right\}}\right]\right\} \\
-\lambda_{n}^{\otimes d}\left\{\prod_{j \in u}\left[\mu^{\prime}\left(x_{j}\right)+x_{j}-1_{\left\{x_{j}>Z_{j}\right\}}\right]\right\} \\
=\left(\lambda^{\otimes d}-\lambda_{n}^{\otimes d}\right)\left\{\prod_{j \in u}\left[\mu^{\prime}\left(x_{j}\right)+x_{j}-1_{\left\{x_{j}>Z_{j}\right\}}\right]\right\} .
\end{aligned}
$$

This helps understanding the forthcoming asymptotic results and justifies this notation that will be used frequently in the following:

$$
g_{u}(\mathbf{x}, \mathbf{z})=\prod_{j \in u}\left[\mu^{\prime}\left(x_{j}\right)+x_{j}-1_{\left\{x_{j}>z_{j}\right\}}\right] .
$$

In the general case of equation (2.1), the choices

$$
\begin{aligned}
\mu(x)=-\frac{1}{2}\left(x^{2}-x+\frac{1}{6}\right), & \beta=2, \quad M=\frac{4}{3} \\
\mu(x)=-\frac{1}{2}\left(\left|x-\frac{1}{2}\right|^{2}-\left|x-\frac{1}{2}\right|+\frac{1}{6}\right), & \beta=1, \quad M=\frac{13}{12}, \\
\mu(x)=\frac{1}{6}-\frac{x^{2}}{2}, & \beta=1, \quad M=\frac{4}{3},
\end{aligned}
$$

yield the symmetric, the centered, and the star discrepancy, respectively (see LFHL01).

${ }^{6}$ The only problem is that, if the weights $\prod_{j \in u} \gamma_{j}$ or $\gamma_{d,|u|}$ are zero for some $u$, then weighted $\mathcal{L}^{p}$-discrepancies can converge to 0 even if the sample $\mathcal{P}_{n}$ is not uniformly scattered. However, if the weights are bounded away from zero, the asymptotic theory is exactly the same. 
In particular, the star $\mathcal{L}^{p}$-discrepancies can be written more simply as

$$
\begin{aligned}
D_{p}^{*}\left(\mathcal{P}_{n}\right) & =\left[\sum_{u \neq \varnothing}\left\|\frac{\left|\mathcal{P}_{n, u} \cap\left[\mathbf{0}, \mathbf{x}_{u}\right)\right|}{n}-\operatorname{Vol}\left(\left[\mathbf{0}, \mathbf{x}_{u}\right)\right)\right\|_{\mathcal{L}^{p}}^{p}\right]^{1 / p} \\
& =\left[\sum_{u \neq \varnothing} \int_{[0,1]^{u}} \frac{\left|\mathcal{P}_{n, u} \cap\left[\mathbf{0}, \mathbf{x}_{u}\right)\right|}{n}-\left.\operatorname{Vol}\left(\left[\mathbf{0}, \mathbf{x}_{u}\right)\right)\right|^{p} \mathrm{~d} \mathbf{x}_{u}\right]^{1 / p} .
\end{aligned}
$$

The special case

$$
D_{\infty}^{*}\left(\mathcal{P}_{n}\right)=\sup _{\mathbf{x} \in[0,1]^{d}}\left|\frac{\left|\mathcal{P}_{n} \cap[\mathbf{0}, \mathbf{x})\right|}{n}-\operatorname{Vol}([\mathbf{0}, \mathbf{x}))\right|
$$

(see, e.g., Hic98a, p. 316, equation (5.1b), or [Nie92, Definition 2.1) is also called star discrepancy (remark that this definition does not coincide with that of [FW94, p. 33, equation (1.4.2)) and coincides with the Kolmogorov-Smirnov statistic for testing uniformity on $[0,1]^{d}$. On the other hand, $D_{2}^{*}\left(\mathcal{P}_{n}\right)$ and $D_{1}^{*}\left(\mathcal{P}_{n}\right)$ yield, respectively, the Cramér-von Mises statistic (see Hic99]) and the $\mathcal{L}^{1}$-test of Schmid and Trede (ST96; see also SW86, p. 149).

It is well known that, when $n \rightarrow+\infty$, the star discrepancy $D_{\infty}^{*}\left(\mathcal{P}_{n}\right)$ converges to 0 if and only if the sample $\mathcal{P}_{n}$ is uniformly scattered. Moreover, under the hypothesis of uniform distribution, $\sqrt{n} D_{\infty}^{*}\left(\mathcal{P}_{n}\right)$ converges in distribution to a well-defined random variable: this means that the average-case error of a Monte Carlo integration procedure decreases (in a certain average sense) as $\frac{1}{\sqrt{n}}$. A worst-case error is given by a Law of the Iterated Logarithm (LIL) for the discrepancy $D_{\infty}^{*}\left(\mathcal{P}_{n}\right)$, stating that

$$
D_{\infty}^{*}\left(\mathcal{P}_{n}\right)=O\left(\sqrt{\frac{\ln \ln n}{n}}\right) \quad \mathbb{P}-\text { as }
$$

([FW94], p. 19). Similar results holds also for the Cramér-von Mises statistics (e.g., the LIL has been proved by [Fin71]).

In this section, we prove that these results also hold for the generalized discrepancies $D_{p}\left(\mathcal{P}_{n}\right)$. Then, we derive the asymptotic distribution of $D_{p}\left(\mathcal{P}_{n}\right)$ as a function of a stochastic integral with respect to a pinned Brownian sheet (for the definitions see, e.g., [AS87, pp. 1345-1346).

Proposition 2.1. Let $\mathcal{P}_{n}$ be given by independent and identically distributed random variables. Then, the following three facts hold:

(i) $D_{p}\left(\mathcal{P}_{n}\right) \rightarrow 0 \mathbb{P}$ - as if and only if $\mathcal{P}_{n}$ is drawn from $\mathbb{P}$ (the uniform measure on $\left.[0,1]^{d}\right)$; on the other hand, $D_{p}\left(\mathcal{P}_{n}\right) \nrightarrow 0 \mathbb{P}^{*}-$ as if and only if $\mathcal{P}_{n}$ is drawn from $\mathbb{P}^{*} \neq \mathbb{P}$.

(ii) Under the probability measure $\mathbb{P}$ (the uniform measure on $[0,1]^{d}$ ),

$$
D_{p}\left(\mathcal{P}_{n}\right)=O_{\mathbb{P}}\left(\frac{1}{\sqrt{n}}\right) .
$$

(iii) Under the probability measure $\mathbb{P}$ (the uniform measure on $[0,1]^{d}$ ), there exists $\mathbb{P}$ - as a finite constant $K>0$ such that $D_{p}\left(\mathcal{P}_{n}\right)$ satisfies the limit inequality

$$
\limsup _{n \rightarrow \infty} \frac{\sqrt{n} D_{p}\left(\mathcal{P}_{n}\right)}{\sqrt{\ln \ln n}} \leq K .
$$


This can be restated saying that the inequality

$$
D_{p}\left(\mathcal{P}_{n}\right)>K \sqrt{\frac{\ln \ln n}{n}}
$$

holds only for a finite number of indexes $n$.

Remark 2.2. In the special case $p=2$, the Law of the Iterated Logarithm could also be proved using the results of Deh89 or GKLZ01.

The following proposition yields an asymptotic distributional result for $\sqrt{n} D_{p}\left(\mathcal{P}_{n}\right)$ under the null hypothesis of uniform distribution of the sample.

Proposition 2.3. Under the probability measure $\mathbb{P}$ (the uniform measure on $\left.[0,1]^{d}\right)$, the statistic $D_{p}\left(\mathcal{P}_{n}\right)$ given by (2.1) has the asymptotic distribution

$$
\sqrt{n} D_{p}\left(\mathcal{P}_{n}\right) \stackrel{\mathcal{D}}{\longrightarrow}\left[\sum_{u \neq \varnothing} \beta^{p|u|} \cdot \int_{[0,1]^{u}}\left|\mathbb{Z}^{|u|}\left(\mathbf{x}_{u}\right)\right|^{p} \mathrm{~d} \mathbf{x}_{u}\right]^{1 / p},
$$

where $\left\{\mathbb{Z}^{|u|}\left(\mathbf{x}_{u}\right), u \subseteq\{1, \ldots, d\}\right\}$ is a collection of centered Gaussian processes indexed by the subset $u \subseteq\{1, \ldots, d\}$. Any $\mathbb{Z}^{|u|}\left(\mathbf{x}_{u}\right)$ is defined as the stochastic integral

$$
\mathbb{Z}^{|u|}\left(\mathbf{x}_{u}\right)=\int_{[0,1]^{d}} g_{u}(\mathbf{x}, \mathbf{y}) \mathrm{d} \mathbb{B}(\mathbf{y})
$$

where $\mathbb{B}$ is a d-dimensional pinned Brownian sheet. The processes $\mathbb{Z}^{|u|}\left(\mathbf{x}_{u}\right)$ are characterized by the covariance functions:

$$
\begin{aligned}
& \operatorname{Cov}\left[\mathbb{Z}^{|u|}\left(\mathbf{x}_{u}\right), \mathbb{Z}^{|u|}\left(\mathbf{z}_{u}\right)\right]= \prod_{j \in u}\left[\mu^{\prime}\left(x_{j}\right) \mu^{\prime}\left(z_{j}\right)-x_{j} z_{j}+x_{j} \vee z_{j}\right] \\
&-\prod_{j \in u}\left[\mu^{\prime}\left(x_{j}\right) \mu^{\prime}\left(z_{j}\right)\right], \\
& \operatorname{Cov}\left[\mathbb{Z}^{\left|u_{1}\right|}\left(\mathbf{x}_{u_{1}}\right), \mathbb{Z}^{\left|u_{2}\right|}\left(\mathbf{z}_{u_{2}}\right)\right]= \prod_{j \in u_{1} \backslash u_{2}} \mu^{\prime}\left(x_{j}\right) \cdot \prod_{j \in u_{2} \backslash u_{1}} \mu^{\prime}\left(z_{j}\right) \\
& \cdot \prod_{j \in u_{1} \cap u_{2}}\left[\mu^{\prime}\left(x_{j}\right) \mu^{\prime}\left(z_{j}\right)-x_{j} z_{j}+x_{j} \vee z_{j}\right] \\
&\left.-\prod_{j \in u_{1} \cap u_{2}} \mu^{\prime}\left(x_{j}\right) \mu^{\prime}\left(z_{j}\right)\right\},
\end{aligned}
$$

for $u, u_{1}, u_{2} \subseteq\{1, \ldots, d\}$.

Remark 2.4. (i) This result can be used as follows to assess uniformity of points. Suppose we observe a set of points $\mathcal{P}_{n}$ believed to come from a uniformly distributed sample. In this case,

$$
\sqrt{n} D_{p}\left(\mathcal{P}_{n}\right) \stackrel{\mathcal{D}}{\longrightarrow}\left[\sum_{u \neq \varnothing} \beta^{p|u|} \cdot \int_{[0,1]^{u}}\left|\mathbb{Z}^{|u|}\left(\mathbf{x}_{u}\right)\right|^{p} \mathrm{~d} \mathbf{x}_{u}\right]^{1 / p}
$$


and

$$
\mathbb{P}\left\{\sqrt{n} D_{p}\left(\mathcal{P}_{n}\right) \leq x\right\} \approx \mathbb{P}\left\{\left[\sum_{u \neq \varnothing} \beta^{p|u|} \cdot \int_{[0,1]^{u}}\left|\mathbb{Z}^{|u|}\left(\mathbf{x}_{u}\right)\right|^{p} \mathrm{~d} \mathbf{x}_{u}\right]^{1 / p} \leq x\right\}
$$

Therefore, if we want to test that $\mathcal{P}_{n}$ is uniform, we verify whether $\sqrt{n} D_{p}\left(\mathcal{P}_{n}\right)$ falls into the interval $\left[0, \gamma_{\alpha}\right]$, where $\gamma_{\alpha}$ is defined by

$$
\begin{aligned}
1-\alpha & =\mathbb{P}\left\{\left[\sum_{u \neq \varnothing} \beta^{p|u|} \cdot \int_{[0,1]^{u}}\left|\mathbb{Z}^{|u|}\left(\mathbf{x}_{u}\right)\right|^{p} \mathrm{~d} \mathbf{x}_{u}\right]^{1 / p} \leq \gamma_{\alpha}\right\} \\
& \approx \mathbb{P}\left\{\sqrt{n} D_{p}\left(\mathcal{P}_{n}\right) \leq \gamma_{\alpha}\right\}
\end{aligned}
$$

and $\alpha$ is $1 \%$ or $5 \%$. In this case, if the points are indeed uniformly distributed, we asymptotically make an error only with probability $\alpha$.

(ii) A particularly interesting case arises when $p=2$. In this case

$$
\begin{aligned}
n\left[D_{p}\left(\mathcal{P}_{n}\right)\right]^{2} & \stackrel{\mathcal{D}}{\longrightarrow} \sum_{u \neq \varnothing} \beta^{2|u|} \cdot \int_{[0,1]^{u}}\left|\mathbb{Z}^{|u|}\left(\mathbf{x}_{u}\right)\right|^{2} \mathrm{~d} \mathbf{x}_{u} \\
& =\sum_{u \neq \varnothing} \beta^{2|u|} \cdot \int_{[0,1]^{u}} \int_{[0,1]^{d}} \int_{[0,1]^{d}} g_{u}(\mathbf{x}, \mathbf{y}) g_{u}(\mathbf{x}, \mathbf{z}) \mathrm{d} \mathbb{B}(\mathbf{y}) \mathrm{d} \mathbb{B}(\mathbf{z}) \mathrm{d} \mathbf{x}_{u} \\
& =\int_{[0,1]^{d}} \int_{[0,1]^{d}}\left\{\sum_{u \neq \varnothing} \beta^{2|u|} \cdot \int_{[0,1]^{u}} g_{u}(\mathbf{x}, \mathbf{y}) g_{u}(\mathbf{x}, \mathbf{z}) \mathrm{d} \mathbf{x}_{u}\right\} \mathrm{d} \mathbb{B}(\mathbf{y}) \mathrm{d} \mathbb{B}(\mathbf{z}) \\
& =\int_{[0,1]^{d}} \int_{[0,1]^{d}} h(\mathbf{y}, \mathbf{z}) \mathrm{d} \mathbb{B}(\mathbf{y}) \mathrm{d} \mathbb{B}(\mathbf{z})
\end{aligned}
$$

where we have set

$$
h(\mathbf{y}, \mathbf{z}) \triangleq \sum_{u \neq \varnothing} \beta^{2|u|} \cdot \int_{[0,1]^{u}} g_{u}(\mathbf{x}, \mathbf{y}) g_{u}(\mathbf{x}, \mathbf{z}) \mathrm{d} \mathbf{x}_{u} .
$$

Since

$$
\begin{aligned}
& 0<\int_{[0,1]^{d}} h(\mathbf{y}, \mathbf{y}) \mathrm{d} \mathbf{y}<\infty, \\
& 0<\int_{[0,1]^{d}} \int_{[0,1]^{d}} h(\mathbf{y}, \mathbf{z}) \mathrm{d} \mathbf{y} \mathrm{d} \mathbf{z}<\infty,
\end{aligned}
$$

the spectrum of the integral operator $\mathcal{A}$ defined as

$$
\mathcal{A} m(\mathbf{y})=\int_{[0,1]^{d}} h(\mathbf{y}, \mathbf{z}) m(\mathbf{z}) \lambda^{\otimes d}(\mathrm{~d} \mathbf{z}),
$$

for $\mathbf{y}, \mathbf{z} \in[0,1]^{d}, m \in L^{2}$, consists of a sequence of nonnegative eigenvalues $\left(\lambda_{j}\right)$ with $\lambda_{1} \geq \lambda_{2} \geq \cdots \geq 0$ and $\sum_{j=1}^{\infty} \lambda_{j}<\infty$, and corresponding eigenvectors $\left(\phi_{j}\right)$ that can be taken orthonormal. Therefore, $h(\mathbf{y}, \mathbf{z})$ can be written as $\sum_{j=1}^{\infty} \lambda_{j} \cdot \phi_{j}(\mathbf{y}) \phi_{j}(\mathbf{z})$ 
in $L^{2}$, and (2.2) becomes

$$
\begin{aligned}
\sum_{u \neq \varnothing} \beta^{2|u|} \cdot \int_{[0,1]^{u}}\left|\mathbb{Z}^{|u|}\left(\mathbf{x}_{u}\right)\right|^{2} \mathrm{~d} \mathbf{x}_{u} & =\sum_{j=1}^{\infty} \lambda_{j} \cdot \int_{[0,1]^{d}} \int_{[0,1]^{d}} \phi_{j}(\mathbf{y}) \phi_{j}(\mathbf{z}) \mathrm{d} \mathbb{B}(\mathbf{y}) \mathrm{d} \mathbb{B}(\mathbf{z}) \\
& =\sum_{j=1}^{\infty} \lambda_{j} \cdot\left(\int_{[0,1]^{d}} \phi_{j}(\mathbf{y}) \mathrm{d} \mathbb{B}(\mathbf{y})\right)^{2} .
\end{aligned}
$$

Now, the quantities defined by $X_{j}=\left(\int_{[0,1]^{d}} \phi_{j}(\mathbf{y}) \mathrm{d} \mathbb{B}(\mathbf{y})\right)$, for $j=1, \ldots, \infty$, are independent standard Gaussian random variables. Therefore, we can write

$$
n\left[D_{p}\left(\mathcal{P}_{n}\right)\right]^{2} \stackrel{\mathcal{D}}{\longrightarrow} \sum_{j=1}^{\infty} \lambda_{j} \cdot X_{j}^{2}
$$

where $\left(\lambda_{j}\right)$ and $\left(X_{j}\right)$ are defined as above. The random variable $\sum_{j=1}^{\infty} \lambda_{j} \cdot X_{j}^{2}$ is a linear combination of chi-squared random variables and is called a second order Gaussian chaos. This result has also been obtained in CS05b in a completely different way (using the properties of degenerate $V$-statistics). The eigenvalues $\left(\lambda_{j}\right)$ and the distribution of the random variable $\sum \lambda_{j} X_{j}^{2}$ can be approximated through the algorithms exposed in CS05a.

\section{AsYmptotic RESUlts FOR $\mathcal{L}^{p}$-DISCREPANCIES UNDER NONUNIFORMITY}

A centered and scaled version of $D_{p}\left(\mathcal{P}_{n}\right)$ converges to a well-defined random variable under the alternative too, that is, when the sample $\mathcal{P}_{n}$ does not come from the uniform distribution on $[0,1]^{d}$. While for $p=\infty$ the asymptotic distribution is very complicated, in the case $p<\infty$ the limit distribution reduces to a normal random variable with a complex variance (see Ang83 for the case of the Cramérvon Mises statistic, i.e., $p=2$ and Rag73 for the case of the Kolmogorov-Smirnov statistic, i.e., $p=\infty)$. We give an explicit formula for the variance and we provide an alternative representation as a function of Brownian sheets.

Proposition 3.1. Under the probability measure $\mathbb{P}^{*} \neq \lambda^{\otimes d}$ (i.e. $\mathbb{P}^{*}$ is not the uniform measure on $[0,1]^{d}$ ), we have

$$
D_{p}\left(\mathcal{P}_{n}\right) \underset{n \rightarrow \infty}{\longrightarrow} D_{p}\left(\mathcal{P}_{\infty}\right), \quad \mathbb{P}^{*}-\text { as }
$$

where

$$
D_{p}\left(\mathcal{P}_{\infty}\right)=\left[\sum_{u \neq \varnothing} \beta^{|u| p} \cdot \int_{[0,1]^{u}}\left|\left(\mathbb{P}-\mathbb{P}^{*}\right) g_{u}(\mathbf{x}, \mathbf{Z})\right|^{p} \mathrm{~d} \mathbf{x}_{u}\right]^{1 / p} .
$$

Proposition 3.2. If $p<\infty$, under the probability measure $\mathbb{P}^{*} \neq \lambda^{\otimes d}$ (i.e. $\mathbb{P}^{*}$ is not the uniform measure on $[0,1]^{d}$ ) with $c d f F^{*}$, we have

$$
\sqrt{n}\left[D_{p}\left(\mathcal{P}_{n}\right)-D_{p}\left(\mathcal{P}_{\infty}\right)\right] \stackrel{\mathcal{D}}{\longrightarrow} \frac{N}{p\left[D_{p}\left(\mathcal{P}_{\infty}\right)\right]^{p-1}},
$$


where $N$ is a centered Gaussian random variable with variance given by

$$
\begin{aligned}
\sigma^{2}= & \mathbb{P}^{*}\left[p \sum_{u \neq \varnothing} \beta^{|u| p} \cdot \int_{[0,1]^{d}}\left[\left(\mathbb{P}-\mathbb{P}^{*}\right) g_{u}(\mathbf{x}, \mathbf{Z})\right]^{p-1} \cdot\left[g_{u}(\mathbf{x}, \mathbf{y})-\mathbb{P}^{*} g_{u}(\mathbf{x}, \mathbf{Y})\right] \mathrm{d} \mathbf{x}_{u}\right]^{2} \\
= & p^{2} \sum_{u \neq \varnothing} \sum_{v \neq \varnothing} \beta^{(|u|+|v|) p} \cdot \int_{[0,1]^{d}} \int_{[0,1]^{d}}\left[\left(\mathbb{P}-\mathbb{P}^{*}\right) g_{u}(\mathbf{x}, \mathbf{Z})\right]^{p-1} \\
& \cdot\left[\left(\mathbb{P}-\mathbb{P}^{*}\right) g_{v}\left(\mathbf{x}, \mathbf{Z}^{\prime}\right)\right]^{p-1} \\
& \cdot\left\{\mathbb{P}^{*}\left[g_{u}(\mathbf{x}, \mathbf{y}) g_{v}(\mathbf{x}, \mathbf{y})\right]-\mathbb{P}^{*} g_{u}(\mathbf{x}, \mathbf{Y}) \mathbb{P}^{*} g_{v}(\mathbf{x}, \mathbf{Y})\right\} \mathrm{d} \mathbf{x}_{u} \mathrm{~d} \mathbf{x}_{v} .
\end{aligned}
$$

$N$ can be expressed alternatively as the following stochastic integral:

$$
N=p \cdot \sum_{u \neq \varnothing} \beta^{|u| p} \cdot \int_{[0,1]^{d}}\left[\left(\mathbb{P}-\mathbb{P}^{*}\right) g_{u}(\mathbf{x}, \mathbf{Z})\right]^{p-1} \cdot \mathbb{Z}_{F^{*}}^{|u|}\left(\mathbf{x}_{u}\right) \mathrm{d} \mathbf{x}_{u}
$$

where

$$
\mathbb{Z}_{F^{*}}^{|u|}\left(\mathbf{x}_{u}\right)=\int_{[0,1]^{d}} g_{u}(\mathbf{x}, \mathbf{y}) \mathrm{d} \mathbb{B}_{F^{*}}(\mathbf{y})
$$

and $\mathbb{B}_{F^{*}}$ is the centered Gaussian process characterized by the variance

$$
\operatorname{Cov}\left[\mathbb{B}_{F^{*}}(\mathbf{x}), \mathbb{B}_{F^{*}}(\mathbf{z})\right]=F^{*}(\mathbf{x} \wedge \mathbf{z})-F^{*}(\mathbf{x}) \cdot F^{*}(\mathbf{z}) .
$$

Remark 3.3. (i) The asymptotic distribution when $p=\infty$ is nonstandard and could be obtained as in Rag73 and SW86] (p. 177).

(ii) We go back to the framework of Remark 2.4(i). Suppose, now, that the points are not uniformly distributed. Then, the probability that $\sqrt{n} D_{p}\left(\mathcal{P}_{n}\right)$ falls into the interval $\left[0, \gamma_{\alpha}\right]$ is approximately given by

$$
\begin{aligned}
& \mathbb{P}\left\{\sqrt{n} D_{p}\left(\mathcal{P}_{n}\right) \in\left[0, \gamma_{\alpha}\right]\right\}=\mathbb{P}\left\{\sqrt{n} D_{p}\left(\mathcal{P}_{n}\right) \leq \gamma_{\alpha}\right\} \\
& \quad=\mathbb{P}\left\{\sqrt{n}\left[D_{p}\left(\mathcal{P}_{n}\right)-D_{p}\left(\mathcal{P}_{\infty}\right)\right] \leq \gamma_{\alpha}-\sqrt{n} D_{p}\left(\mathcal{P}_{\infty}\right)\right\} \\
& \quad \approx \Phi\left(\frac{\gamma_{\alpha}-\sqrt{n} D_{p}\left(\mathcal{P}_{\infty}\right)}{\sigma}\right),
\end{aligned}
$$

where $\sigma^{2}$ is the asymptotic variance of $\sqrt{n}\left[D_{p}\left(\mathcal{P}_{n}\right)-D_{p}\left(\mathcal{P}_{\infty}\right)\right]$. Since $D_{p}\left(\mathcal{P}_{\infty}\right)>$ 0 , as $n \rightarrow \infty$, we get

$$
\Phi\left(\frac{\gamma_{\alpha}-\sqrt{n} D_{p}\left(\mathcal{P}_{\infty}\right)}{\sigma}\right) \rightarrow 0
$$

and the test rejects the null hypothesis with probability converging to 1 .

(iii) Equation (3.1) can be used to derive the variance of the Cramér-von Mises statistic under the alternative, as in Ang83. Since the Cramér-von Mises statistic is the star discrepancy with $p=2$ and $d=1$, we have $\beta=1, M=\frac{4}{3}$ and $g_{1}(x, z)=-1_{\{x>z\}}$, and we recover the formulas (stated for a general choice of $\mathbb{P}$ ) on page 2480 of Ang83.

\section{A simulation study}

In the following we will show some of the previous results using the generalized $\mathcal{L}^{2}$-discrepancies as an example. We just consider the $\mathcal{L}^{2}$-case since this is the framework of [LFHL01]. We will let $n \in\{25,50,100,200,400\}$ and $d \in\{1,2,5\}, 7$

\footnotetext{
${ }^{7}$ The simulations have been performed using Ox Professional 3.0 (see [Doo01]).
} 
4.1. Finite sample distribution of $n \cdot\left[D_{2}\left(\mathcal{P}_{n}\right)\right]^{2}$. The following simulation study shows some characteristics of $n \cdot\left[D_{2}\left(\mathcal{P}_{n}\right)\right]^{2}$ for several sample sizes. For every graphic, we have drawn 10,000 times a sample $\mathcal{P}_{n}$ of size $n$ of uniform independent random variables on $[0,1]^{d}$. We have calculated $n \cdot\left[D_{2}\left(\mathcal{P}_{n}\right)\right]^{2}$ for each of the 10,000 samples and for the three statistics proposed by [Hic98a]; that is, the centered, the star and the symmetric one. Then we have represented the density (as a histogram and a kernel estimator) and the $Q-Q$ plot with respect to a Gaussian random variable with the same mean and the same variance (see Figures 4.1, 4.2, 4.3, 4.4, 4.5. 4.6 and 4.7). For $d=1$, we have represented just the centered discrepancy since the others are equal up to a scalar multiplication, the constant being 1 for the star and 4 for the symmetric discrepancy.

For $n$ varying and $d$ fixed, the distribution of $n \cdot\left[D_{2}\left(\mathcal{P}_{n}\right)\right]^{2}$ is remarkably stable: apart from some fluctuations in the upper tail of the distribution, the overall form is almost the same, especially when the behavior is compared with what happens under the alternative hypothesis of nonuniformity. Moreover, another feature of the finite sample distributions is the fact that, as long as $d$ increases, the distribution of $n \cdot\left[D_{2}\left(\mathcal{P}_{n}\right)\right]^{2}$ appears to be less skewed: this is compatible with the fact that, when both $n$ and $d$ go to infinity at suitable rates, the asymptotic distribution of the scaled discrepancy is a Gaussian distribution. These two stylized facts are reflected by the results of [CS05b], for the case of $\mathcal{L}^{2}$-discrepancies and related quantities.
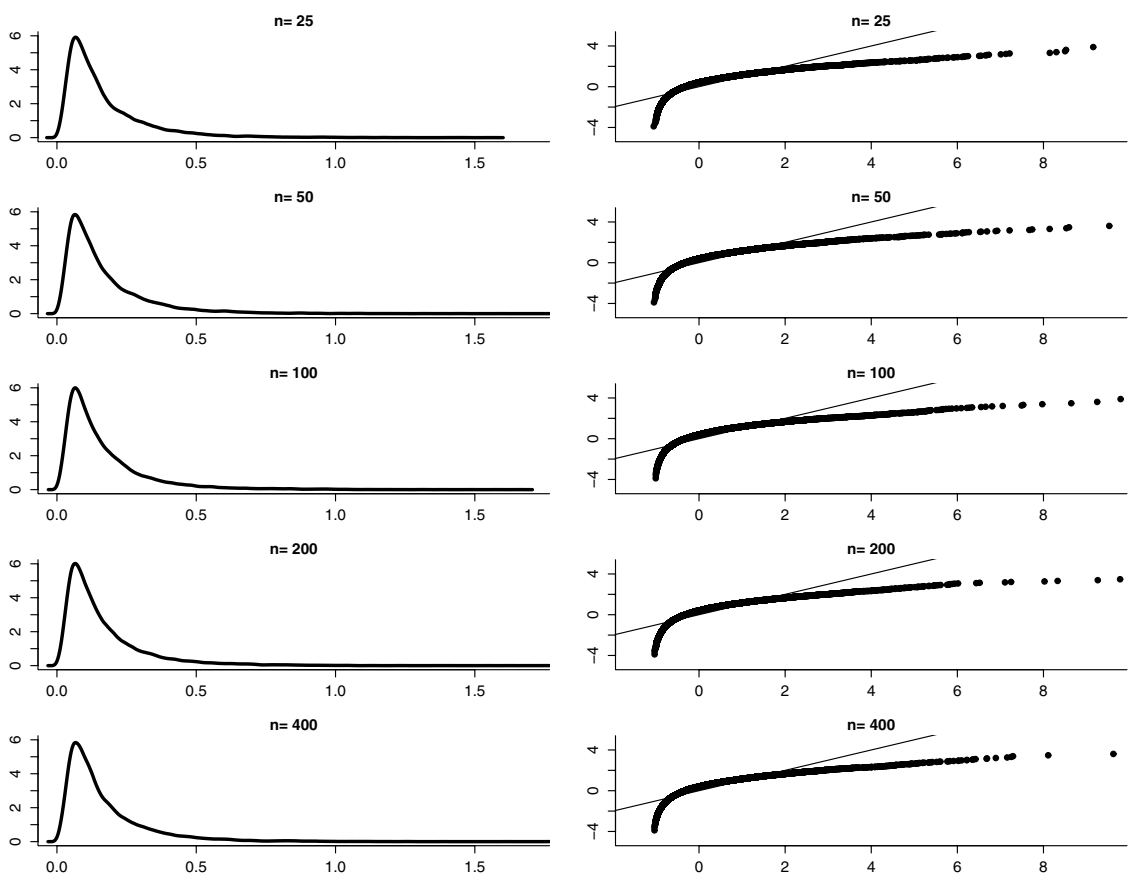

Figure 4.1. Centered $n \cdot\left[D_{2}\left(\mathcal{P}_{n}\right)\right]^{2}$ for $d=1$ 

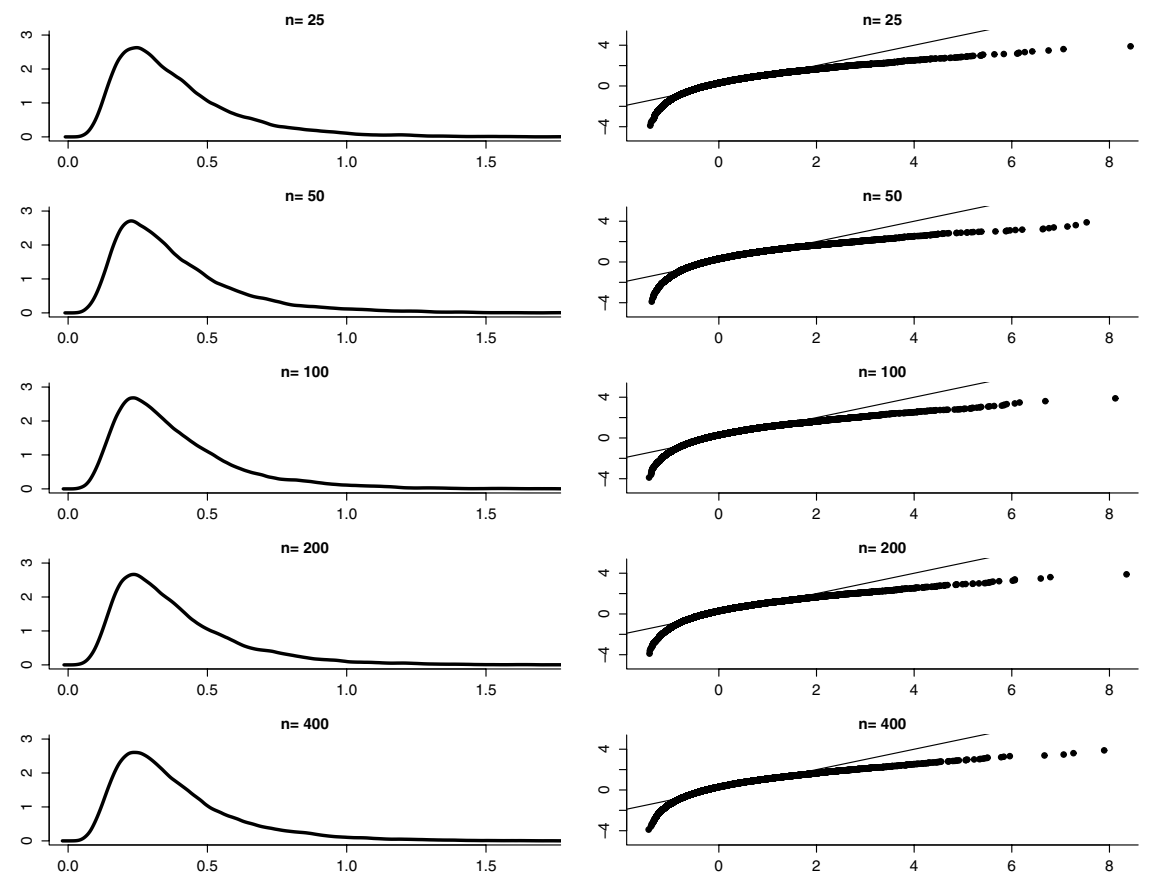

Figure 4.2. Centered $n \cdot\left[D_{2}\left(\mathcal{P}_{n}\right)\right]^{2}$ for $d=2$
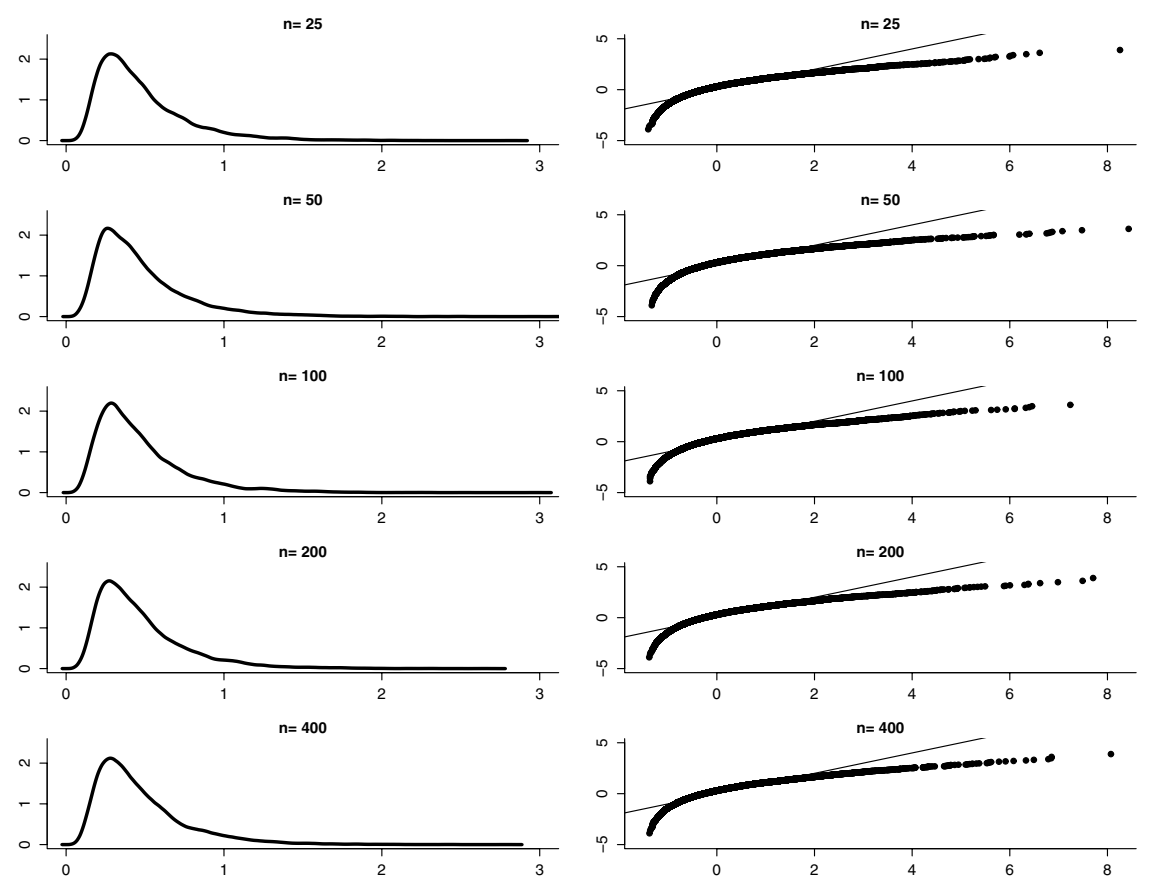

FIgURE 4.3. Star $n \cdot\left[D_{2}\left(\mathcal{P}_{n}\right)\right]^{2}$ for $d=2$ 

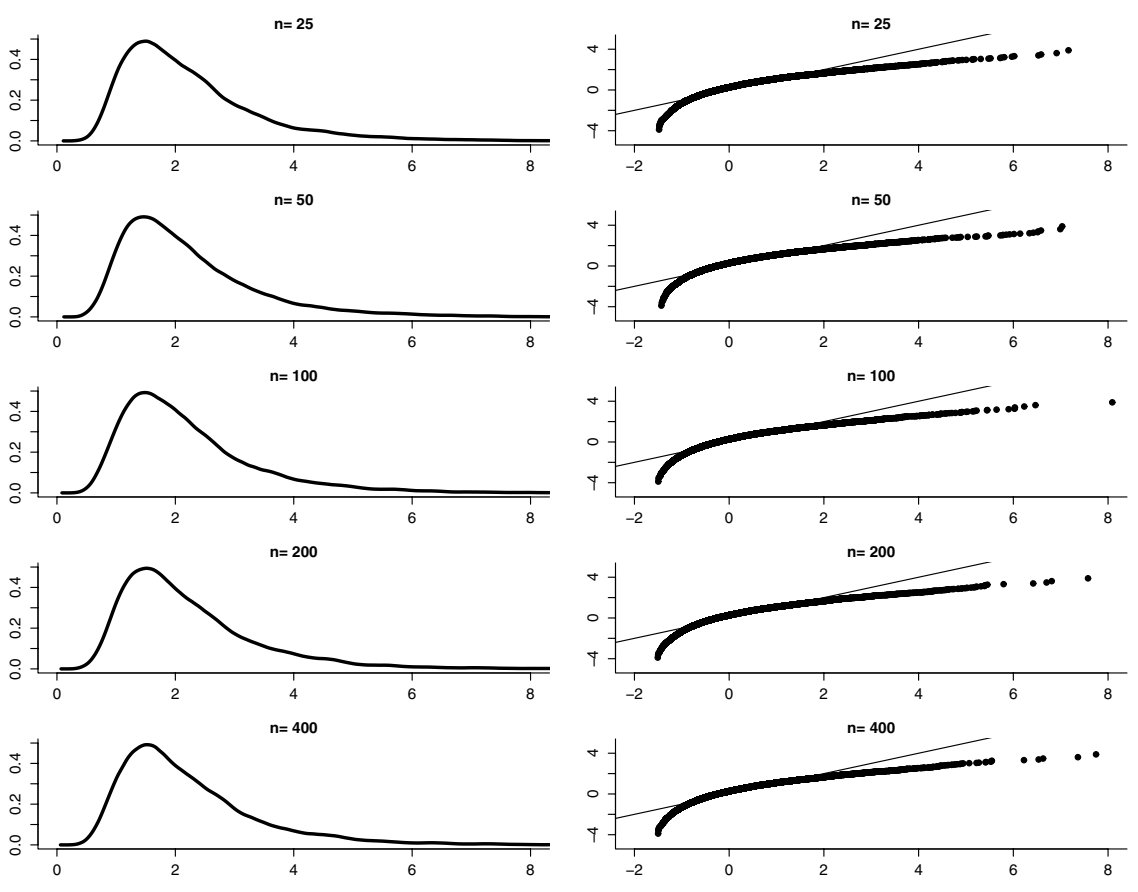

FiguRE 4.4. Symmetric $n \cdot\left[D_{2}\left(\mathcal{P}_{n}\right)\right]^{2}$ for $d=2$
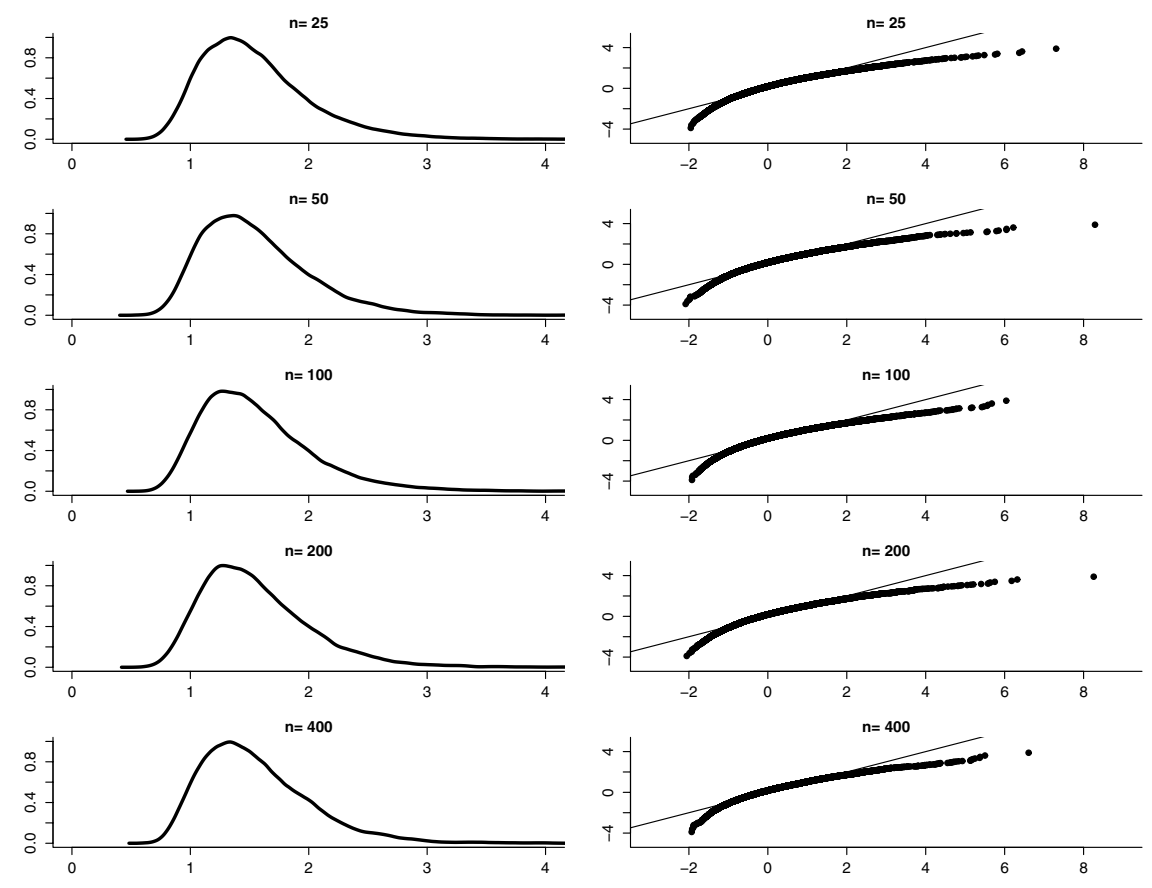

FiguRE 4.5. Centered $n \cdot\left[D_{2}\left(\mathcal{P}_{n}\right)\right]^{2}$ for $d=5$ 

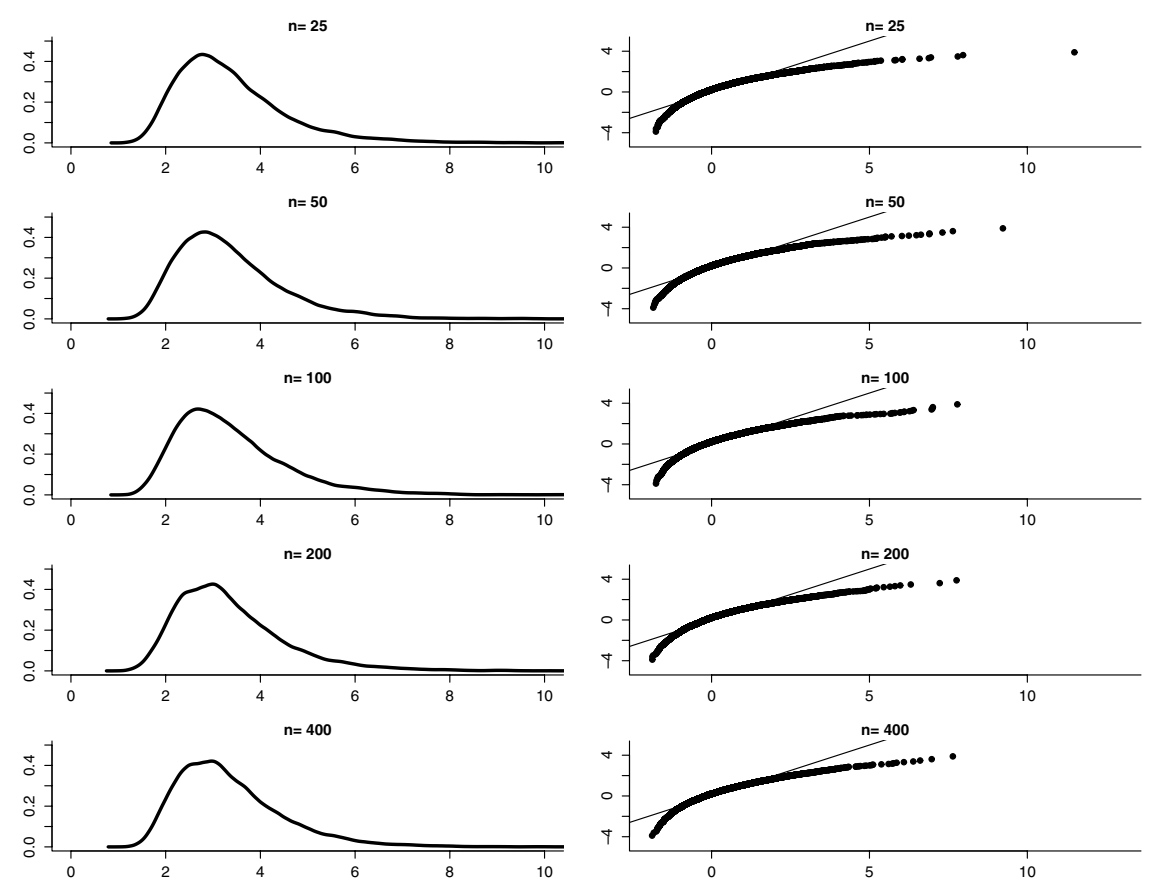

Figure 4.6. Star $n \cdot\left[D_{2}\left(\mathcal{P}_{n}\right)\right]^{2}$ for $d=5$
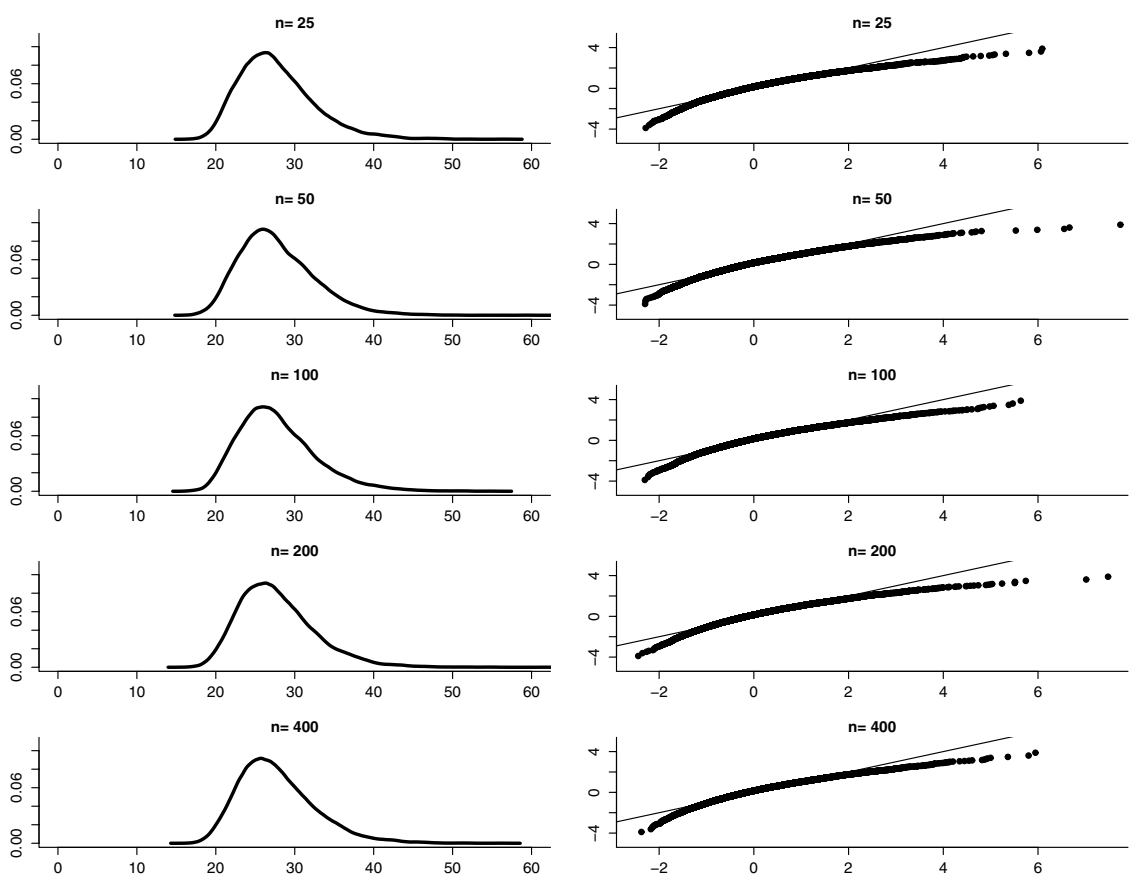

Figure 4.7. Symmetric $n \cdot\left[D_{2}\left(\mathcal{P}_{n}\right)\right]^{2}$ for $d=5$ 
4.2. $\left[D_{2}\left(\mathcal{P}_{n}\right)\right]^{2}$ under the alternative. The following simulated example shows that $\left[D_{2}\left(\mathcal{P}_{n}\right)\right]^{2}$ approaches a normal distribution when the points are not uniformly distributed on $[0,1]^{d}$ (see Figure 4.8). For each graphic, we have drawn 10,000 samples $\mathcal{P}_{n}$ of size $n$ of $\operatorname{Beta}(2,2)$ independent random variables on $[0,1]$. Therefore, we expect to have

$$
\sqrt{n}\left(\frac{\left[D_{2}\left(\mathcal{P}_{n}\right)\right]^{2}-.004762}{.01810}\right) \stackrel{\mathcal{D}}{\rightarrow} \mathcal{N}(0,1)
$$

The graphics are similar to the previous ones, but we have subtracted the true mean from the values of the discrepancies, divided by the true standard deviation and multiplied by $\sqrt{n}$. We have represented only the centered discrepancy, since the others are equal up to a scalar multiplication.

The convergence towards a Gaussian random variable is evident, but slower than the convergence towards a second order Gaussian Chaos under the null (this is compatible with the convergence rates expressed by the Berry-Esséen bounds of CS05b).
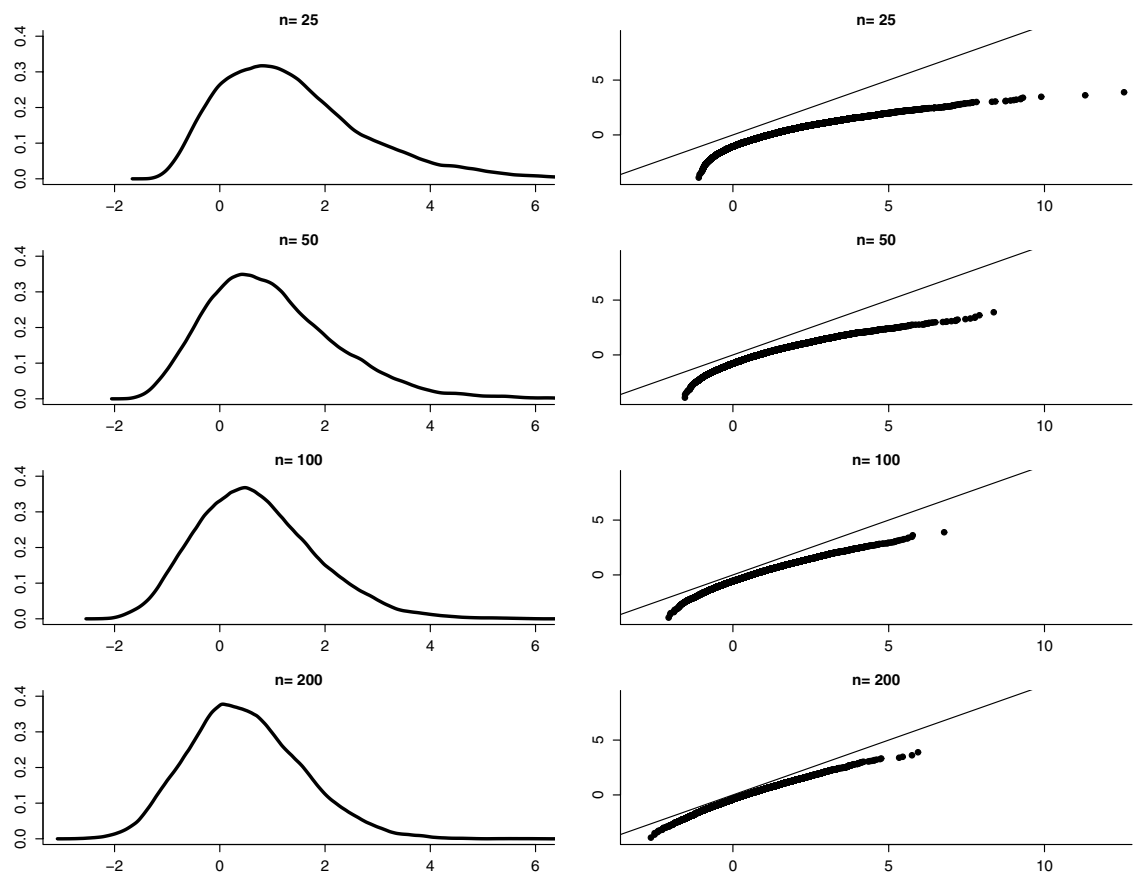

Figure 4.8. Convergence of $\left[D_{2}\left(\mathcal{P}_{n}\right)\right]^{2}$ towards a normal random variable 


\section{Proofs}

Proof of Proposition 2.1. We start with a general majorization result for $D_{p}\left(\mathcal{P}_{n}\right)$ that will be used several times in the remainder of the proof. Using Theorem 5.2 in Hic98a, it is possible to show that any $\mathcal{L}^{p}$-discrepancy $D_{p}\left(\mathcal{P}_{n}\right)$ can be majorized by a constant $K$ times the corresponding $\mathcal{L}^{p}$-star discrepancy $D_{p}^{*}\left(\mathcal{P}_{n}\right)$ (see Hic98a, Section 5.1, for a definition). Indeed, taking $\beta_{1}=\beta, \mu_{1}=\mu, \beta_{2}=1, \mu_{2}=\frac{1}{6}-\frac{x^{2}}{2}$, we have

$$
D_{p}\left(\mathcal{P}_{n}\right) \leq K\left(q, 1, \beta, \frac{1}{6}-\frac{x^{2}}{2}, \mu\right) \cdot D_{p}^{*}\left(\mathcal{P}_{n}\right)
$$

where $q^{-1}+p^{-1}=1$. Moreover, from the fact that $\mu \in X_{\infty}$ (see Hic98a, equation $(2.4)), \mu^{\prime}$ is bounded and $K=K\left(q, 1, \beta, \frac{1}{6}-\frac{x^{2}}{2}, \mu\right)$ is finite. Writing $D_{p}^{*}\left(\mathcal{P}_{n}\right)$ as in Hic98a, (page 316, equation 5.1a), we have

$$
\left[D_{p}^{*}\left(\mathcal{P}_{n}\right)\right]^{p}=\sum_{u \neq \varnothing}\left\|\frac{\left|\mathcal{P}_{n, u} \cap\left[\mathbf{0}, \mathbf{x}_{u}\right)\right|}{n}-\operatorname{Vol}\left(\left[\mathbf{0}, \mathbf{x}_{u}\right)\right)\right\|_{\mathcal{L}^{p}}^{p},
$$

where $\mathcal{P}_{n, u}$ denotes the projection of the sample $\mathcal{P}_{n}$ on the unit cube $[0,1]^{u}$. Therefore, from Lyapunov's inequality, $\left\|\frac{\left|\mathcal{P}_{n, u} \cap\left[\mathbf{0}, \mathbf{x}_{u}\right)\right|}{n}-\operatorname{Vol}\left(\left[\mathbf{0}, \mathbf{x}_{u}\right)\right)\right\|_{\mathcal{L}^{p}}$ can be majorized by the $\mathcal{L}^{\infty}$ distance. This leads to the majorization formula

$$
D_{p}\left(\mathcal{P}_{n}\right) \leq K \cdot D_{p}^{*}\left(\mathcal{P}_{n}\right) \leq K \cdot\left\{\sum_{u \neq \varnothing}\left[D_{\infty}^{*}\left(\mathcal{P}_{n, u}\right)\right]^{p}\right\}^{\frac{1}{p}}
$$

Using this result, the first part of (i) is trivial, since $\mathcal{P}_{n, u}$ is a uniform sample on $[0,1]^{u}$, for any $u$, and the Glivenko-Cantelli Theorem implies that $D_{\infty}^{*}\left(\mathcal{P}_{n, u}\right) \rightarrow 0$ almost surely. As concerns the second part of (i), using Theorem 5.2 in Hic98a it is possible to show that any $\mathcal{L}^{p}$-discrepancy $D_{p}\left(\mathcal{P}_{n}\right)$ can be minorized by a constant times the corresponding $\mathcal{L}^{p}$-star discrepancy $D_{p}^{*}\left(\mathcal{P}_{n}\right)$ (see Hic98a], Section 5.1, for a definition). Indeed, taking $\beta_{1}=1, \mu_{1}=\frac{1}{6}-\frac{x^{2}}{2}, \beta_{2}=\beta, \mu_{2}=\mu$, we have

$$
D_{p}^{*}\left(\mathcal{P}_{n}\right) \leq K\left(q, \beta, 1, \mu, \frac{1}{6}-\frac{x^{2}}{2}\right) \cdot D_{p}\left(\mathcal{P}_{n}\right)
$$

where $q^{-1}+p^{-1}=1 . K\left(q, \beta, 1, \mu, \frac{1}{6}-\frac{x^{2}}{2}\right)$ is strictly positive since it is greater than $\max \left\{\beta, \beta^{s}\right\}$. Moreover, the generalized $\mathcal{L}^{p}$-star discrepancy can be minorized as $D_{p}^{*}\left(\mathcal{P}_{n}\right) \geq\left\|\frac{\left|\mathcal{P}_{n} \cap[\mathbf{0}, \mathbf{x})\right|}{n}-\operatorname{Vol}([\mathbf{0}, \mathbf{x}))\right\|_{\mathcal{L}^{p}}$, and the latter converges to a strictly positive constant as long as $n \rightarrow \infty$.

To prove (ii), we just need to show that for any $\delta$ there exists a $\lambda$ such that

$$
\sup _{n \in \mathbb{N}} \mathbb{P}\left\{\sqrt{n} D_{p}\left(\mathcal{P}_{n}\right) \geq \lambda\right\} \leq \delta
$$


This derives from the application of (5.2)

$$
\begin{aligned}
\mathbb{P}\left\{D_{p}\left(\mathcal{P}_{n}\right) \geq \frac{\lambda}{\sqrt{n}}\right\} & \leq \mathbb{P}\left\{\sum_{u \neq \varnothing}\left[D_{\infty}^{*}\left(\mathcal{P}_{n, u}\right)\right]^{p} \geq\left(\frac{\lambda}{K \sqrt{n}}\right)^{p}\right\} \\
& \leq \sum_{u \neq \varnothing} \mathbb{P}\left\{\left[D_{\infty}^{*}\left(\mathcal{P}_{n, u}\right)\right]^{p} \geq \frac{1}{\left(2^{d}-1\right)}\left(\frac{\lambda}{K \sqrt{n}}\right)^{p}\right\} \\
& \leq \sum_{u \neq \varnothing} \mathbb{P}\left\{D_{\infty}^{*}\left(\mathcal{P}_{n, u}\right) \geq \frac{m}{\sqrt{n}}\right\}
\end{aligned}
$$

where we have set $m=\lambda /\left[\left(2^{d}-1\right)^{\frac{1}{p}} K\right]$. Here, an inequality of Kiefer and Wolfowitz (see [KW58) (indeed a multivariate extension of the Dvoretzky-KieferWolfowitz or DKW inequality) implies the existence of positive constants $c$ and $c^{\prime}$ such that

$$
\mathbb{P}\left\{D_{\infty}^{*}\left(\mathcal{P}_{n, u}\right) \geq \frac{m}{\sqrt{n}}\right\} \leq c^{\prime} \cdot \exp \left\{-c m^{2}\right\}
$$

for all $n>0, m \geq 0$ and $u$. Therefore, from (5.3) and (5.4)

$$
\mathbb{P}\left\{D_{p}\left(\mathcal{P}_{n}\right) \geq \frac{\lambda}{\sqrt{n}}\right\} \leq c^{\prime} \cdot\left(2^{d}-1\right) \cdot \exp \left\{-\frac{c \lambda^{2}}{\left(2^{d}-1\right)^{\frac{2}{p}} K^{2}}\right\},
$$

and the RHS is independent of $n$.

As concerns (iii), recall that $\sqrt{n} \cdot D_{\infty}^{*}\left(\mathcal{P}_{n, u}\right)$ is exactly the empirical process of the sample $\mathcal{P}_{n, u}$ on $[0,1]^{u}$ and then, by the LIL (see, e.g., Kie61, FW94, p. 19) we have

$$
\limsup _{n \rightarrow \infty} \frac{\sqrt{n} \cdot D_{\infty}^{*}\left(\mathcal{P}_{n, u}\right)}{\sqrt{\ln \ln n}} \leq \frac{1}{\sqrt{2}} .
$$

This yields the result.

Proof of Proposition 2.3. First of all, we recall the definitions of the empirical measures $\lambda_{n}^{\otimes d}$ and $\lambda_{n}^{\otimes|u|}$ for $u \subseteq\{1, \ldots, d\}$ and the associated integrals:

$$
\begin{array}{cc}
\lambda_{n}^{\otimes d}=\frac{1}{n} \sum_{\mathbf{z} \in \mathcal{P}_{n}} \delta_{\mathbf{z}}, & \lambda_{n}^{\otimes|u|}=\frac{1}{n} \sum_{\mathbf{z} \in \mathcal{P}_{n}} \delta_{\mathbf{z}_{u}}, \\
\lambda_{n}^{\otimes d} f=\int f \mathrm{~d} \lambda_{n}^{\otimes d}=\frac{1}{n} \sum_{\mathbf{z} \in \mathcal{P}_{n}} f(\mathbf{z}), & \lambda_{n}^{\otimes|u|} f=\int f \mathrm{~d} \lambda_{n}^{\otimes|u|}=\frac{1}{n} \sum_{\mathbf{z} \in \mathcal{P}_{n}} f\left(\mathbf{z}_{u}\right),
\end{array}
$$

where $\delta_{\mathbf{z}}$ is the Dirac measure in $\mathbf{z}$ and $\mathbf{z}_{u}$ is the projection of $\mathbf{z}$ on $[0,1]^{u}$. Recalling that

$$
\prod_{j \in u} \mu^{\prime}\left(x_{j}\right)=\lambda^{\otimes d} g_{u}(\mathbf{x}, \mathbf{Z})=\int_{[0,1]^{d}} g_{u}(\mathbf{x}, \mathbf{z}) \mathrm{d} \mathbf{z},
$$

(2.1) can be written as

$$
D_{p}\left(\mathcal{P}_{n}\right)=\left[\sum_{u \neq \varnothing} \beta^{p|u|} \cdot \int_{[0,1]^{d}}\left|\left(\lambda^{\otimes d}-\lambda_{n}^{\otimes d}\right) g_{u}(\mathbf{x}, \mathbf{Z})\right|^{p} \mathrm{~d} \mathbf{x}\right]^{1 / p} .
$$


Since, by Proposition 2.1(ii) $\sqrt{n} D_{p}\left(\mathcal{P}_{n}\right)=O_{\mathbb{P}}(1)$, (15.6) becomes

$$
\sqrt{n} D_{p}\left(\mathcal{P}_{n}\right)=\left[\sum_{u \neq \varnothing} \beta^{p|u|} \cdot \int_{[0,1]^{d}}\left|\sqrt{n}\left(\lambda^{\otimes d}-\lambda_{n}^{\otimes d}\right) g_{u}(\mathbf{x}, \mathbf{Z})\right|^{p} \mathrm{~d} \mathbf{x}\right]^{1 / p},
$$

and we are led to consider the asymptotic behavior of the empirical process (see vdV98, p. 266)

$$
\mathbb{Z}_{n}^{|u|}\left(\mathbf{x}_{u}\right)=\sqrt{n}\left(\lambda^{\otimes d}-\lambda_{n}^{\otimes d}\right) g_{u}(\mathbf{x}, \mathbf{Z}) .
$$

We need to show that, uniformly in $\mathbf{x} \in[0,1]^{d}$ and for any $u \subseteq\{1, \ldots, d\}$, $\mathbb{Z}_{n}^{|u|}\left(\mathbf{x}_{u}\right)$ converges in the space of bounded functions towards a stochastic integral with respect to a $|u|$-dimensional pinned Brownian sheet. In order to do so, we show that the class of functions

$$
\mathcal{F}=\left\{g_{u}(\mathbf{x}, \cdot), \text { for any } \mathbf{x} \in[0,1]^{d}, u \subseteq\{1, \ldots, d\}\right\}
$$

is $\lambda^{\otimes d}$-Donsker (see vdVW96, p. 81). The elements of $\mathcal{F}$ are linear combinations of a finite number of elements of the form

$$
\prod_{j \in u_{1}}\left(\mu^{\prime}\left(x_{j}\right)+x_{j}\right) \cdot \prod_{j \in u_{2}} 1_{\left\{x_{j}>\cdot j\right\}}
$$

for $u_{1} \cap u_{2}=\varnothing$ and $u_{1} \cup u_{2}=u \subseteq\{1, \ldots, d\}$. From Example 2.1.3 in vdVW96],

$$
\left\{\prod_{j \in u_{2}} 1_{\left\{x_{j}>\cdot{ }_{j}\right\}}, \mathbf{x}_{u_{2}} \in[0,1]^{u_{2}}, u_{2} \subseteq\{1, \ldots, d\}\right\}
$$

is a $\lambda^{\otimes d}$-Donsker class; the property is preserved by multiplication with the class

$$
\left\{\prod_{j \in u_{1}}\left(\mu^{\prime}\left(x_{j}\right)+x_{j}\right), \mathbf{x}_{u_{1}} \in[0,1]^{u_{1}}, u_{1} \subseteq\{1, \ldots, d\}\right\}
$$

(we remark that $\mu^{\prime}(\cdot) \in \mathcal{L}^{\infty}([0,1])$ and is therefore bounded by a constant) and by linear combination.

Therefore, $\mathbb{Z}_{n}^{|u|}\left(\mathbf{x}_{u}\right)$ converges uniformly over $\mathcal{F}$ to the stated limit, and simple manipulations show that $\mathbb{Z}^{|u|}\left(\mathbf{x}_{u}\right)$ can be expressed as

$$
\mathbb{Z}^{|u|}\left(\mathbf{x}_{u}\right)=\int_{[0,1]^{d}} g_{u}(\mathbf{x}, \mathbf{y}) \mathrm{d} \mathbb{B}(\mathbf{y}),
$$

where $\mathbb{B}$ denotes a pinned Brownian sheet (the multi-dimensional analogue of a Brownian bridge). The Gaussian process $\mathbb{Z}^{|u|}\left(\mathbf{x}_{u}\right)$ is characterized by the same mean and covariance of $\mathbb{Z}_{n}^{|u|}\left(\mathbf{x}_{u}\right)$.

Now, for finite $p$, the function

$$
\left\|\left(f_{u}\right)\right\|: f \mapsto\left[\sum_{u \neq \varnothing} \beta^{p|u|} \int_{[0,1]^{d}}\left|f_{u}\right|^{p} \mathrm{~d} \mathbf{x}\right]^{1 / p}
$$

([Hic98a , p. 300) is continuous, and therefore the result is derived from the Continuous Mapping Theorem (see Kal97, Theorem 3.27). When $p=\infty$, on the other hand, we have to apply the Argmax Continuous Mapping Theorem (vdV98, Corollary 5.58). The limiting process has continuous sample paths, and maxima of 
Gaussian processes are unique by Lemma 2.6 in KP90. As concerns the uniform tightness of the maximum of $\left(\mathbb{Z}_{n}^{|u|}\left(\mathbf{x}_{u}\right)\right)_{n}$, it means that

$$
\sup _{\mathbf{x}}\left|\sqrt{n}\left(\lambda^{\otimes d}-\lambda_{n}^{\otimes d}\right) g_{u}(\mathbf{x}, \mathbf{Y})\right|=O_{\mathbb{P}}(1)
$$

that is, for any $\varepsilon$ there exists an $M$ such that

$$
\sup _{n \in \mathbb{N}} \mathbb{P}\left\{\sup _{\mathbf{x}}\left|\sqrt{n}\left(\lambda^{\otimes d}-\lambda_{n}^{\otimes d}\right) g_{u}(\mathbf{x}, \mathbf{Y})\right|>M\right\} \leq \varepsilon
$$

First we decompose $g_{u}(\mathbf{x}, \mathbf{y})$ as

$$
\begin{aligned}
g_{u}(\mathbf{x}, \mathbf{y}) & =\prod_{j \in u}\left[\mu^{\prime}\left(x_{j}\right)+x_{j}-1_{\left\{x_{j}>y_{j}\right\}}\right] \\
& =\sum_{u^{\prime} \subseteq u}\left\{\prod_{j \in u \backslash u^{\prime}} \mu^{\prime}\left(x_{j}\right)\right\} \cdot\left\{\prod_{j \in u^{\prime}}\left[x_{j}-1_{\left\{x_{j}>y_{j}\right\}}\right]\right\},
\end{aligned}
$$

and we write

$$
\begin{aligned}
& \sup _{\mathbf{x}}\left|\sqrt{n}\left(\lambda^{\otimes d}-\lambda_{n}^{\otimes d}\right) g_{u}(\mathbf{x}, \mathbf{Y})\right| \\
& =\sup _{\mathbf{x}}\left|\sum_{u^{\prime} \subseteq u}\left\{\prod_{j \in u \backslash u^{\prime}} \mu^{\prime}\left(x_{j}\right)\right\} \cdot \sqrt{n}\left(\lambda^{\otimes d}-\lambda_{n}^{\otimes d}\right)\left\{\prod_{j \in u^{\prime}}\left[x_{j}-1_{\left\{x_{j}>y_{j}\right\}}\right]\right\}\right| \\
& \leq \sum_{u^{\prime} \subseteq u} \sup _{\mathbf{x}_{u \backslash u^{\prime}}}\left|\prod_{j \in u \backslash u^{\prime}} \mu^{\prime}\left(x_{j}\right)\right| \cdot \sup _{\mathbf{x}_{u^{\prime}}}\left|\sqrt{n}\left(\lambda^{\otimes d}-\lambda_{n}^{\otimes d}\right)\left\{\prod_{j \in u^{\prime}}\left[x_{j}-1_{\left\{x_{j}>y_{j}\right\}}\right]\right\}\right| \\
& \leq \sum_{u^{\prime} \subseteq u} m^{\left|u \backslash u^{\prime}\right|} .\left\|\sqrt{n}\left(\lambda^{\otimes d}-\lambda_{n}^{\otimes d}\right)\left\{\prod_{j \in u^{\prime}}\left[x_{j}-1_{\left\{x_{j}>y_{j}\right\}}\right]\right\}\right\|_{\mathcal{L}^{\infty}},
\end{aligned}
$$

where we have used the fact that $\sup _{x_{j}}\left|\mu^{\prime}\left(x_{j}\right)\right| \leq m$. Therefore (5.7) becomes

$$
\begin{aligned}
& \sup _{n \in \mathbb{N}} \mathbb{P}\left\{\sup _{\mathbf{x}}\left|\sqrt{n}\left(\lambda^{\otimes d}-\lambda_{n}^{\otimes d}\right) g_{u}(\mathbf{x}, \mathbf{Y})\right|>M\right\} \\
& \leq \sup _{n \in \mathbb{N}} \mathbb{P}\left\{\sum_{u^{\prime} \subseteq u} m^{\left|u \backslash u^{\prime}\right|} .\left\|\sqrt{n}\left(\lambda^{\otimes d}-\lambda_{n}^{\otimes d}\right)\left\{\prod_{j \in u^{\prime}}\left[x_{j}-1_{\left\{x_{j}>y_{j}\right\}}\right]\right\}\right\|_{\mathcal{L}^{\infty}}>M\right\} \\
& \leq \sup _{n \in \mathbb{N}} \sum_{u^{\prime} \subseteq u} \mathbb{P}\left\{\left\|\sqrt{n}\left(\lambda^{\otimes d}-\lambda_{n}^{\otimes d}\right)\left\{\prod_{j \in u^{\prime}}\left[\cdot_{j}-1_{\left\{\cdot_{j}>y_{j}\right\}}\right]\right\}\right\|_{\mathcal{L}^{\infty}}>\frac{M}{\left.2^{|u|} m^{\left|u \backslash u^{\prime}\right|}\right\} .}\right.
\end{aligned}
$$


Using inequality (5.4), we get

$$
\begin{aligned}
\sup _{n \in \mathbb{N}} \mathbb{P} & \left\{\sup _{\mathbf{x}}\left|\sqrt{n}\left(\lambda^{\otimes d}-\lambda_{n}^{\otimes d}\right) g_{u}(\mathbf{x}, \mathbf{Y})\right|>M\right\} \\
& \leq \sup _{n \in \mathbb{N}} \sum_{u^{\prime} \subseteq u} \mathbb{P}\left\{\left\|\left(\lambda^{\otimes d}-\lambda_{n}^{\otimes d}\right)\left\{\prod_{j \in u^{\prime}}\left[x_{j}-1_{\left\{x_{j}>\cdot \cdot_{j}\right\}}\right]\right\}\right\|_{\mathcal{L}^{\infty}}>\frac{M}{2^{|u|} m^{\left|u \backslash u^{\prime}\right|} \sqrt{n}}\right\} \\
& \leq \sum_{u^{\prime} \subseteq u} c^{\prime} \cdot \exp \left\{-c \cdot \frac{M^{2}}{\left.2^{2|u|} m^{2\left|u \backslash u^{\prime}\right|}\right\}}\right. \\
& \leq c^{\prime} \cdot 2^{|u|} \cdot \exp \left\{-c \cdot \frac{M^{2}}{2^{2|u|} \max \left\{1, m^{2|u|}\right\}}\right\} .
\end{aligned}
$$

Setting

$$
M=\frac{2^{|u|} \max \left\{1, m^{|u|}\right\}}{\sqrt{c}} \cdot \sqrt{\ln \left(\frac{2^{|u|} c^{\prime}}{\varepsilon}\right)}
$$

we get the uniform tightness required.

Proof of Proposition 3.1. The proof is trivial and uses repeated application of the as version of the Slutsky Theorem (see [Dav94, p. 286, Theorem 18.8(i)), starting from the fact that, under the alternative, $\mathbb{P}^{*}$-almost surely:

$$
\begin{aligned}
\frac{1}{n} \sum_{\mathbf{z} \in \mathcal{P}_{n}} g_{u}(\mathbf{x}, \mathbf{z}) & =\mathbb{P}_{n}^{*} g_{u}(\mathbf{x}, \mathbf{Z}) \\
& \underset{n \rightarrow \infty}{\longrightarrow} \int_{[0,1]^{d}} g_{u}(\mathbf{x}, \mathbf{z}) \mathrm{d} \mathbb{P}^{*}(\mathbf{z})=\mathbb{P}^{*} g_{u}(\mathbf{x}, \mathbf{Z}) .
\end{aligned}
$$

Proof of Proposition 3.2. Our strategy of proof is to link the asymptotic distribution of $D_{p}\left(\mathcal{P}_{n}\right)$ to that of $\left[D_{p}\left(\mathcal{P}_{n}\right)\right]^{p}$ using the standard delta method, then to express the asymptotic distribution of $\left[D_{p}\left(\mathcal{P}_{n}\right)\right]^{p}$ in terms of an empirical process using the functional delta method (see vdV98, Chapter 20) and then to derive the asymptotic distribution of this empirical process. We divide the proof into six steps.

(1) We rewrite (2.1) as

$$
D_{p}\left(\mathcal{P}_{n}\right)=\left[\sum_{u \neq \varnothing} \beta^{|u| p} \cdot \int_{[0,1]^{u}}\left|\prod_{j \in u} \mu^{\prime}\left(x_{j}\right)-Z\left(\mathbf{x}_{u} ; \mathbb{P}_{n}^{*}\right)\right|^{p} \mathrm{~d} \mathbf{x}_{u}\right]^{1 / p},
$$

where

$$
\begin{aligned}
Z\left(\mathbf{x}_{u} ; \mathbb{P}_{n}^{*}\right) & =\frac{1}{n} \sum_{\mathbf{z} \in \mathcal{P}_{n}} g_{u}(\mathbf{x}, \mathbf{z}) \\
& =\int_{[0,1]^{d}} g_{u}(\mathbf{x}, \mathbf{z}) \mathrm{d} \mathbb{P}_{n}^{*}(\mathbf{z}) .
\end{aligned}
$$


From Proposition [3.1] as long as $n \rightarrow \infty, D_{p}\left(\mathcal{P}_{n}\right)$ converges $\mathbb{P}^{*}$-almost surely to $D_{p}\left(\mathcal{P}_{\infty}\right)$ with $Z\left(\mathbf{x}_{u} ; \mathbb{P}^{*}\right)=\int_{[0,1]^{d}} g_{u}(\mathbf{x}, \mathbf{z}) \mathrm{d} \mathbb{P}^{*}(\mathbf{z})$ :

$$
D_{p}\left(\mathcal{P}_{\infty}\right)=\left[\sum_{u \neq \varnothing} \beta^{|u| p} \cdot \int_{[0,1]^{u}}\left|\prod_{j \in u} \mu^{\prime}\left(x_{j}\right)-Z\left(\mathbf{x}_{u} ; \mathbb{P}^{*}\right)\right|^{p} \mathrm{~d} \mathbf{x}_{u}\right]^{1 / p} .
$$

We remark that $Z\left(\mathbf{x}_{u} ; \mathbb{P}_{n}^{*}\right)$ is a random perturbation of $Z\left(\mathbf{x}_{u} ; \mathbb{P}^{*}\right)$ and

$$
\sqrt{n}\left[Z\left(\mathbf{x}_{u} ; \mathbb{P}_{n}^{*}\right)-Z\left(\mathbf{x}_{u} ; \mathbb{P}^{*}\right)\right]=\sqrt{n}\left(\mathbb{P}_{n}^{*}-\mathbb{P}^{*}\right) g_{u}(\mathbf{x}, \mathbf{Z})
$$

is an empirical process.

(2) First of all, applying the standard delta method to the function $\phi(x)=x^{p}$, we get

$$
\begin{aligned}
& \sqrt{n}\left(\left[D_{p}\left(\mathcal{P}_{n}\right)\right]^{p}-\left[D_{p}\left(\mathcal{P}_{\infty}\right)\right]^{p}\right) \\
& \quad=\sqrt{n} p\left[D_{p}\left(\mathcal{P}_{\infty}\right)\right]^{p-1} \cdot\left(D_{p}\left(\mathcal{P}_{n}\right)-D_{p}\left(\mathcal{P}_{\infty}\right)\right)+o_{\mathbb{P}}(1), \\
& \sqrt{n}\left(D_{p}\left(\mathcal{P}_{n}\right)-D_{p}\left(\mathcal{P}_{\infty}\right)\right) \\
& \quad=\frac{\sqrt{n}\left(\left[D_{p}\left(\mathcal{P}_{n}\right)\right]^{p}-\left[D_{p}\left(\mathcal{P}_{\infty}\right)\right]^{p}\right)}{p\left[D_{p}\left(\mathcal{P}_{\infty}\right)\right]^{p-1}}+o_{\mathbb{P}}(1)
\end{aligned}
$$

Therefore, we are led to study the behavior of $\left(\left[D_{p}\left(\mathcal{P}_{n}\right)\right]^{p}-\left[D_{p}\left(\mathcal{P}_{\infty}\right)\right]^{p}\right)$. Using (5.8) and (5.9),$\sqrt{n}\left(\left[D_{p}\left(\mathcal{P}_{n}\right)\right]^{p}-\left[D_{p}\left(\mathcal{P}_{\infty}\right)\right]^{p}\right)$ becomes

$$
\begin{aligned}
\sqrt{n}\left(\left[D_{p}\left(\mathcal{P}_{n}\right)\right]^{p}-\left[D_{p}\left(\mathcal{P}_{\infty}\right)\right]^{p}\right) & \\
=\sum_{u \neq \varnothing} \beta^{|u| p} \cdot \sqrt{n} \int_{[0,1]^{u}}\left\{\left|\prod_{j \in u} \mu^{\prime}\left(x_{j}\right)-Z\left(\mathbf{x}_{u} ; \mathbb{P}_{n}^{*}\right)\right|^{p}\right. & \\
& \left.-\left|\prod_{j \in u} \mu^{\prime}\left(x_{j}\right)-Z\left(\mathbf{x}_{u} ; \mathbb{P}^{*}\right)\right|^{p}\right\} \mathrm{d} \mathbf{x}_{u} .
\end{aligned}
$$

(3) Then, we use the functional delta method (see vdV98, Chapter 20]) to approximate the previous formula. Define the statistical functional $\phi_{u}$ as

$$
\phi_{u}(G)=\int_{[0,1]^{u}}\left|\prod_{j \in u} \mu^{\prime}\left(x_{j}\right)-G\left(\mathbf{x}_{u}\right)\right|^{p} \mathrm{~d} \mathbf{x}_{u} .
$$

The difference $\phi_{u}\left(Z\left(\mathbf{x}_{u} ; \mathbb{P}^{*}\right)\right)-\phi_{u}\left(Z\left(\mathbf{x}_{u} ; \mathbb{P}_{n}^{*}\right)\right)$ appears in (5.11), and it is interesting to approximate it through a simpler one (the objective is to obtain a functional that is linear in $\left(\mathbb{P}_{n}^{*}-\mathbb{P}^{*}\right)$; that is, an empirical process). The right way to simplify this formula is to show that this functional is Hadamard differentiable at $G=Z\left(\mathrm{x}_{u} ; \mathbb{P}^{*}\right)$, and the same holds for any $u \neq \varnothing 8$ Therefore, we have to show

${ }^{8}$ We say that map $\phi: \mathbb{D}_{\phi} \rightarrow \mathbb{E}$, defined on a subset $\mathbb{D}_{\phi}$ of a normed space $\mathbb{D}$ containing $\theta$, is Hadamard differentiable at $\theta$ if there exists a continuous, linear map $\phi_{\theta}^{\prime}: \mathbb{D} \rightarrow \mathbb{E}$ such that

$$
\left\|\frac{\phi\left(\theta+t h_{t}\right)-\phi(\theta)}{t}-\phi_{\theta}^{\prime}(h)\right\|_{\mathbb{E}} \rightarrow 0
$$

as $t \downarrow 0$ and for every $h_{t} \rightarrow h$ (such that $\theta+t h_{t}$ is contained in the domain of $\phi$ for all small $t>0$ ). Loosely speaking, this means that the difference $\frac{1}{t} \cdot\left[\phi\left(\theta+t h_{t}\right)-\phi(\theta)\right]$ can be approximated by the linear function $\phi_{\theta}^{\prime}(h)$, whose asymptotic behavior is often much simpler to study. 
that, for every $g_{t} \rightarrow g$, when $t \downarrow 0$, there exists a function $\phi_{u}^{\prime}(g)$ such that

$$
\left\|\frac{\phi_{u}\left(G+t \cdot g_{t}\right)-\phi_{u}(G)}{t}-\phi_{u}^{\prime}(g)\right\| \rightarrow 0 .
$$

In this formula, the reader can identify

$$
\begin{gathered}
t=\frac{1}{\sqrt{n}} \\
G\left(\mathbf{x}_{u}\right)=Z\left(\mathbf{x}_{u} ; \mathbb{P}^{*}\right), \\
\left(G+t \cdot g_{t}\right)\left(\mathbf{x}_{u}\right)=Z\left(\mathbf{x}_{u} ; \mathbb{P}_{n}^{*}\right), \\
g_{t}\left(\mathbf{x}_{u}\right)=\sqrt{n}\left[Z\left(\mathbf{x}_{u} ; \mathbb{P}_{n}^{*}\right)-Z\left(\mathbf{x}_{u} ; \mathbb{P}^{*}\right)\right] .
\end{gathered}
$$

Moreover, $\|\cdot\|$ is the uniform norm $\|\cdot\|_{\mathcal{L}^{\infty}}$ and

$$
\phi_{u}^{\prime}(g)=-p \cdot \int_{[0,1]^{u}}\left(\prod_{j \in u} \mu^{\prime}\left(x_{j}\right)-G\left(\mathbf{x}_{u}\right)\right)^{p-1} g\left(\mathbf{x}_{u}\right) \mathrm{d} \mathbf{x}_{u} .
$$

Now, $\phi_{u}\left(Z\left(\mathbf{x}_{u} ; \mathbb{P}^{*}\right)\right)-\phi_{u}\left(Z\left(\mathbf{x}_{u} ; \mathbb{P}_{n}^{*}\right)\right)$ becomes

$$
\begin{aligned}
\int_{[0,1]^{u}} & \left\{\left|\prod_{j \in u} \mu^{\prime}\left(x_{j}\right)-Z\left(\mathbf{x}_{u} ; \mathbb{P}_{n}^{*}\right)\right|^{p}\right. \\
& \left.-\left|\prod_{j \in u} \mu^{\prime}\left(x_{j}\right)-Z\left(\mathbf{x}_{u} ; \mathbb{P}^{*}\right)\right|^{p}\right\} \mathrm{d} \mathbf{x}_{u} \\
= & \int_{[0,1]^{u}}\left|\prod_{j \in u} \mu^{\prime}\left(x_{j}\right)-\left(G+t \cdot g_{t}\right)\left(\mathbf{x}_{u}\right)\right|^{p} \mathrm{~d} \mathbf{x}_{u} \\
& -\int_{[0,1]^{u}}\left|\prod_{j \in u} \mu^{\prime}\left(x_{j}\right)-G\left(\mathbf{x}_{u}\right)\right|^{p} \mathrm{~d} \mathbf{x}_{u} \\
= & \int_{[0,1]^{u}}\left|\prod_{j \in u} \mu^{\prime}\left(x_{j}\right)-G\left(\mathbf{x}_{u}\right)\right|^{p} \\
& \cdot\left[\left|1-\frac{\left(t \cdot g_{t}\right)\left(\mathbf{x}_{u}\right)}{\left(\prod_{j \in u} \mu^{\prime}\left(x_{j}\right)-G\left(\mathbf{x}_{u}\right)\right)}\right|^{p}-1\right] \mathrm{d} \mathbf{x}_{u} \\
= & \int_{[0,1]^{u}}\left|\prod_{j \in u} \mu^{\prime}\left(x_{j}\right)-G\left(\mathbf{x}_{u}\right)\right|^{p} \\
& \cdot\left[\left(1-\frac{\left(t \cdot g_{t}\right)\left(\mathbf{x}_{u}\right)}{\left(\prod_{j \in u} \mu^{\prime}\left(x_{j}\right)-G\left(\mathbf{x}_{u}\right)\right)}\right)^{p}-1\right] \mathrm{d} \mathbf{x}_{u},
\end{aligned}
$$

where we take $t$ small enough to have

$$
\left(t \cdot g_{t}\right)\left(\mathbf{x}_{u}\right) \leq\left(\prod_{j \in u} \mu^{\prime}\left(x_{j}\right)-G\left(\mathbf{x}_{u}\right)\right) .
$$


Now, using (5.13), (5.12) can be majorized as

$$
\begin{aligned}
& \| \frac{\int_{[0,1]^{u}}\left\{\left|\prod_{j \in u} \mu^{\prime}\left(x_{j}\right)-\left(G+t \cdot g_{t}\right)\left(\mathbf{x}_{u}\right)\right|^{p}-\left|\prod_{j \in u} \mu^{\prime}\left(x_{j}\right)-G\left(\mathbf{x}_{u}\right)\right|^{p}\right\} \mathrm{d} \mathbf{x}_{u}}{t} \\
& +p \cdot \int_{[0,1]^{u}}\left(\prod_{j \in u} \mu^{\prime}\left(x_{j}\right)-G\left(\mathbf{x}_{u}\right)\right)^{p-1} g\left(\mathbf{x}_{u}\right) \mathrm{d} \mathbf{x}_{u} \| \\
& =\| \int_{[0,1]^{u}}\left|\prod_{j \in u} \mu^{\prime}\left(x_{j}\right)-G\left(\mathbf{x}_{u}\right)\right|^{p} \\
& \cdot t^{-1}\left[\left(1-\frac{\left(t \cdot g_{t}\right)\left(\mathbf{x}_{u}\right)}{\left(\prod_{j \in u} \mu^{\prime}\left(x_{j}\right)-G\left(\mathbf{x}_{u}\right)\right)}\right)^{p}-1\right] \mathrm{d} \mathbf{x}_{u} \\
& +p \cdot \int_{[0,1]^{u}}\left(\prod_{j \in u} \mu^{\prime}\left(x_{j}\right)-G\left(\mathbf{x}_{u}\right)\right)^{p-1} g\left(\mathbf{x}_{u}\right) \mathrm{d} \mathbf{x}_{u} \| \\
& =\| \int_{[0,1]^{u}}\left|\prod_{j \in u} \mu^{\prime}\left(x_{j}\right)-G\left(\mathbf{x}_{u}\right)\right|^{p} \\
& \cdot t^{-1}\left(-p \frac{\left(t \cdot g_{t}\right)\left(\mathbf{x}_{u}\right)}{\left(\prod_{j \in u} \mu^{\prime}\left(x_{j}\right)-G\left(\mathbf{x}_{u}\right)\right)}+\mathcal{E}_{t}(u, \mathbf{x})\right) \mathrm{d} \mathbf{x}_{u} \\
& +p \cdot \int_{[0,1]^{u}}\left(\prod_{j \in u} \mu^{\prime}\left(x_{j}\right)-G\left(\mathbf{x}_{u}\right)\right)^{p-1} g\left(\mathbf{x}_{u}\right) \mathrm{d} \mathbf{x}_{u} \| \\
& =\| p \cdot \int_{[0,1]^{u}}\left(\prod_{j \in u} \mu^{\prime}\left(x_{j}\right)-G\left(\mathbf{x}_{u}\right)\right)^{p-1} \cdot\left(g\left(\mathbf{x}_{u}\right)-g_{t}\left(\mathbf{x}_{u}\right)\right) \mathrm{d} \mathbf{x}_{u} \\
& +t^{-1} \cdot \int_{[0,1]^{u}}\left|\prod_{j \in u} \mu^{\prime}\left(x_{j}\right)-G\left(\mathbf{x}_{u}\right)\right|^{p} \cdot \mathcal{E}_{t}(u, \mathbf{x}) \mathrm{d} \mathbf{x}_{u}|| \\
& \leq p \cdot\left\|\int_{[0,1]^{u}}\left|\prod_{j \in u} \mu^{\prime}\left(x_{j}\right)-G\left(\mathbf{x}_{u}\right)\right|^{p-1} \cdot\left|g\left(\mathbf{x}_{u}\right)-g_{t}\left(\mathbf{x}_{u}\right)\right| \mathrm{d} \mathbf{x}_{u}\right\| \\
& +\left\|t^{-1} \cdot \int_{[0,1]^{u}}\left|\prod_{j \in u} \mu^{\prime}\left(x_{j}\right)-G\left(\mathbf{x}_{u}\right)\right|^{p} \cdot \mathcal{E}_{t}(u, \mathbf{x}) \mathrm{d} \mathbf{x}_{u}\right\|,
\end{aligned}
$$

where $\mathcal{E}_{t}$ is defined as

$$
\mathcal{E}_{t}(u, \mathbf{x}) \triangleq\left(1-\frac{\left(t \cdot g_{t}\right)\left(\mathbf{x}_{u}\right)}{\left(\prod_{j \in u} \mu^{\prime}\left(x_{j}\right)-G\left(\mathbf{x}_{u}\right)\right)}\right)^{p}-1+p \frac{\left(t \cdot g_{t}\right)\left(\mathbf{x}_{u}\right)}{\left(\prod_{j \in u} \mu^{\prime}\left(x_{j}\right)-G\left(\mathbf{x}_{u}\right)\right)} .
$$


Now, (5.14) converges to 0 , since

$$
\begin{aligned}
& \left\|\int_{[0,1]^{u}}\left|\prod_{j \in u} \mu^{\prime}\left(x_{j}\right)-G\left(\mathbf{x}_{u}\right)\right|^{p-1} \cdot\left|g\left(\mathbf{x}_{u}\right)-g_{t}\left(\mathbf{x}_{u}\right)\right| \mathrm{d} \mathbf{x}_{u}\right\| \\
& \quad \leq\left\|\int_{[0,1]^{u}}\left|\prod_{j \in u} \mu^{\prime}\left(x_{j}\right)-G\left(\mathbf{x}_{u}\right)\right|^{p-1} \mathrm{~d} \mathbf{x}_{u}\right\| \cdot\left\|g\left(\mathbf{x}_{u}\right)-g_{t}\left(\mathbf{x}_{u}\right)\right\| \rightarrow 0
\end{aligned}
$$

and

$$
\begin{aligned}
\| t^{-1} & \cdot \int_{[0,1]^{u}}\left|\prod_{j \in u} \mu^{\prime}\left(x_{j}\right)-G\left(\mathbf{x}_{u}\right)\right|^{p} \cdot \mathcal{E}_{t} \mathrm{~d} \mathbf{x}_{u} \| \\
& \leq\left\|\int_{[0,1]^{u}}\left|\prod_{j \in u} \mu^{\prime}\left(x_{j}\right)-G\left(\mathbf{x}_{u}\right)\right|^{p} \mathrm{~d} \mathbf{x}_{u}\right\| \cdot t^{-1}\left\|\mathcal{E}_{t}(u, \mathbf{x})\right\| \\
& \rightarrow 0 .
\end{aligned}
$$

This means that (5.11) becomes

$$
\begin{aligned}
& \sqrt{n}\left(\left[D_{p}\left(\mathcal{P}_{n}\right)\right]^{p}-\left[D_{p}\left(\mathcal{P}_{\infty}\right)\right]^{p}\right) \\
& =\sum_{u \neq \varnothing} \beta^{|u| p} \cdot \sqrt{n}\left[\phi_{u}\left(Z\left(\mathbf{x}_{u} ; \mathbb{P}_{n}^{*}\right)\right)-\phi_{u}\left(Z\left(\mathbf{x}_{u} ; \mathbb{P}^{*}\right)\right)\right] \\
& =\sum_{u \neq \varnothing} \beta^{|u| p} \cdot \sqrt{n}\left[\phi _ { u } \left(Z\left(\mathbf{x}_{u} ; \mathbb{P}^{*}\right)\right.\right. \\
& \left.\left.\quad+\frac{\sqrt{n}\left[Z\left(\mathbf{x}_{u} ; \mathbb{P}_{n}^{*}\right)-Z\left(\mathbf{x}_{u} ; \mathbb{P}^{*}\right)\right]}{\sqrt{n}}\right)-\phi_{u}\left(Z\left(\mathbf{x}_{u} ; \mathbb{P}^{*}\right)\right)\right] \\
& =-p \cdot \sum_{u \neq \varnothing} \beta^{|u| p} \cdot \int_{[0,1]^{u}}\left(\prod_{j \in u} \mu^{\prime}\left(x_{j}\right)-Z\left(\mathbf{x}_{u} ; \mathbb{P}^{*}\right)\right)^{p-1} \\
& \cdot \sqrt{n}\left[Z\left(\mathbf{x}_{u} ; \mathbb{P}_{n}^{*}\right)-Z\left(\mathbf{x}_{u} ; \mathbb{P}^{*}\right)\right] \mathrm{d} \mathbf{x}_{u}+o_{\mathbb{P}}(1) .
\end{aligned}
$$

(4) Let $F^{*}$ be the cdf of $\mathbb{P}^{*}$. Then, we show that

$$
\sqrt{n}\left(Z\left(\mathbf{x}_{u} ; \mathbb{P}_{n}^{*}\right)-Z\left(\mathbf{x}_{u} ; \mathbb{P}^{*}\right)\right) \stackrel{\mathcal{D}}{\longrightarrow} \mathbb{Z}_{F^{*}}^{|u|}\left(\mathbf{x}_{u}\right),
$$

for any $u \subseteq\{1, \ldots, d\}$, where

$$
\mathbb{Z}_{F^{*}}^{|u|}\left(\mathbf{x}_{u}\right)=\int_{[0,1]^{d}} g_{u}(\mathbf{x}, \mathbf{y}) \mathrm{dB}_{F^{*}}(\mathbf{y}),
$$

and $\mathbb{B}_{F^{*}}$ is the centered Gaussian process characterized by variance:

$$
\operatorname{Cov}\left[\mathbb{B}_{F^{*}}(\mathbf{x}), \mathbb{B}_{F^{*}}(\mathbf{z})\right]=F^{*}(\mathbf{x} \wedge \mathbf{z})-F^{*}(\mathbf{x}) \cdot F^{*}(\mathbf{z}) .
$$

The method is the same used in the proof of Proposition 2.3 with the same class of functions $\mathcal{F}$ and, indeed, $\mathbb{Z}^{|u|}$ can be obtained setting $\mathbb{P}^{*}=\lambda^{\otimes d}$.

(5) Combining (5.10), (5.15) and (5.16), we get the final distribution. 
(6) The variance of $N$ can be computed, substituting the expression of $\mathbb{Z}_{F^{*}}^{|u|}\left(\mathbf{x}_{u}\right)$ into $N$ and exchanging the order of integration:

$$
\begin{aligned}
N=p \cdot \sum_{u \neq \varnothing} \beta^{|u| p} \cdot \int_{[0,1]^{d}}\left[\left(\mathbb{P}-\mathbb{P}^{*}\right) g_{u}(\mathbf{x}, \mathbf{Z})\right]^{p-1} \\
\cdot\left[\int_{[0,1]^{d}} g_{u}(\mathbf{x}, \mathbf{y}) \mathrm{d} \mathbb{B}_{F^{*}}(\mathbf{y})\right] \mathrm{d} \mathbf{x}_{u} \\
=\int_{[0,1]^{d}}\left[p \sum_{u \neq \varnothing} \beta^{|u| p}\right. \\
\left.\cdot \int_{[0,1]^{d}}\left[\left(\mathbb{P}-\mathbb{P}^{*}\right) g_{u}(\mathbf{x}, \mathbf{Z})\right]^{p-1} \cdot g_{u}(\mathbf{x}, \mathbf{y}) \mathrm{d} \mathbf{x}_{u}\right] \mathrm{dB}_{F^{*}}(\mathbf{y}) .
\end{aligned}
$$

This is a stochastic integral with respect to a Gaussian process, and $\mathbb{V}(N)$ can be computed as in vdV98, p. 269.

\section{REFERENCES}

[Ang83] J.E. Angus, On the asymptotic distribution of Cramér-von Mises one-sample test statistics under an alternative, Comm. Statist. A-Theory Methods 12 (1983), no. 21, 2477-2482. MR0715177 (85c:62040)

[AS87] R.J. Adler and G. Samorodnitsky, Tail behaviour for the suprema of Gaussian processes with applications to empirical processes, Ann. Probab. 15 (1987), no. 4, 13391351. MR0905335 (88j:60073)

[CS05a] C. Choirat and R. Seri, The asymptotic distribution of quadratic discrepancies, Monte Carlo and quasi-Monte Carlo methods 2004, 61-76 (D. Talay and H. Niederreiter, eds.), Springer-Verlag, 2006. MR.2208702 (2006k:65006)

[CS05b] Statistical properties of quadratic discrepancies, Working paper (2005).

[Dav94] J. Davidson, Stochastic limit theory, Advanced Texts in Econometrics, The Clarendon Press, Oxford University Press, New York, 1994. MR.1430804 (97k:60002)

[Deh89] H. Dehling, Complete convergence of triangular arrays and the law of the iterated logarithm for U-statistics, Statist. Probab. Lett. 7 (1989), no. 4, 319-321. MR0980708 (90h:60028)

[Doo01] J.A. Doornik, Ox: An object-oriented matrix language, 4th ed., Timberlake Consultants Press, London, 2001.

[Fin71] H. Finkelstein, The law of the iterated logarithm for empirical distributions, Ann. Math. Statist. 42 (1971), 607-615. MR0287600 (44:4803)

[FMW02] K.-T. Fang, C.-X. Ma, and P. Winker, Centered $L_{2}$-discrepancy of random sampling and Latin hypercube design, and construction of uniform designs, Math. Comp. 71 (2002), no. 237, 275-296 (electronic). MR1863000 (2002h:65024)

[FW94] K.-T. Fang and Y. Wang, Number-theoretic methods in statistics, Monographs on Statistics and Applied Probability, vol. 51, Chapman \& Hall, London, 1994. MR1284470 (95g:65189)

[GKLZ01] E. Giné, S. Kwapień, R. Latała, and J. Zinn, The LIL for canonical U-statistics of order 2, Ann. Probab. 29 (2001), no. 1, 520-557. MR.1825163 (2002k:60083)

[HHW03] H.S. Hong, F.J. Hickernell, and G. Wei, The distribution of the discrepancy of scrambled digital $(t, m, s)$-nets, Math. Comput. Simulation 62 (2003), no. 3-6, 335-345. MR.1988381(2004e:11080)

[Hic96] F.J. Hickernell, Quadrature error bounds with applications to lattice rules, SIAM J. Numer. Anal. 33 (1996), no. 5, 1995-2016. MR.1411860 (97m:65050)

[Hic97] - Erratum: "Quadrature error bounds with applications to lattice rules" [SIAM J. Numer. Anal. 33 (1996), no. 5, 1995-2016;], SIAM J. Numer. Anal. 34 (1997), no. 2, 853-866. MR.1442941(2000h:65042)

[Hic98a] _ A generalized discrepancy and quadrature error bound, Math. Comp. 67 (1998), no. 221, 299-322. MR1433265 (98c:65032) 
[Hic98b] L Lattice rules: how well do they measure up?, Random and quasi-random point sets, Lecture Notes in Statist., vol. 138, Springer, New York, 1998, pp. 109-166. MR1662841 (2000b:65007)

[Hic99] , Goodness-of-fit statistics, discrepancies and robust designs, Statist. Probab. Lett. 44 (1999), no. 1, 73-78. MR.1706366

[HJK98] J. Hoogland, F. James, and R. Kleiss, Quasi-Monte Carlo, discrepancies and error estimates, Monte Carlo and quasi-Monte Carlo methods 1996 (Salzburg), Lecture Notes in Statist., vol. 127, Springer, New York, 1998, pp. 266-276. MR.1644525 (99d:65070)

[HK96a] J. Hoogland and R. Kleiss, Discrepancy-based error estimates for quasi-Monte Carlo. I: General formalism, Comput. Phys. Comm. 98 (1996), no. 1-2, 111-127.

[HK96b] - Discrepancy-based error estimates for quasi-Monte Carlo. II: Results in one dimension, Comput. Phys. Comm. 98 (1996), no. 1-2, 128-136.

[HK97] _ Discrepancy-based error estimates for quasi-Monte Carlo. III: Error distribution and central limits, Comput. Phys. Comm. 101 (1997), no. 1-2, 21-30.

[JHK97] F. James, J. Hoogland, and R. Kleiss, Multidimensional sampling for simulation and integration: Measures, discrepancies and quasi-random numbers, Comput. Phys. Comm. 99 (1997), no. 2-3, 180-220.

[Kal97] O. Kallenberg, Foundations of modern probability, Probability and its Applications (New York), Springer-Verlag, New York, 1997. MR1464694 (99e:60001)

[Kie61] J. Kiefer, On large deviations of the empiric D. F. of vector chance variables and a law of the iterated logarithm, Pacific J. Math. 11 (1961), 649-660. MR0131885 (24:A1732)

[KP90] J. Kim and D. Pollard, Cube root asymptotics, Ann. Statist. 18 (1990), no. 1, 191-219. MR1041391 (91f:62059)

[KW58] J. Kiefer and J. Wolfowitz, On the deviations of the empiric distribution function of vector chance variables, Trans. Amer. Math. Soc. 87 (1958), 173-186. MR0099075 (20:5519)

[Lee96a] H. Leeb, The asymptotic distribution of diaphony in one dimension, GERAD - École des Hautes Études Commerciales, Montréal (1996).

[Lee96b] _ A weak law for diaphony, RIST++, Research Institute for Software Technology, University of Salzburg (1996).

[Lee02] - Asymptotic properties of the spectral test, diaphony, and related quantities, Math. Comp. 71 (2002), no. 237, 297-309 (electronic). MR1863001 (2002j:11086)

[LFHL01] J.-J. Liang, K.-T. Fang, F.J. Hickernell, and R. Li, Testing multivariate uniformity and its applications, Math. Comp. 70 (2001), no. 233, 337-355. MR1680903 (2001f:62032)

[LP03] G. Leobacher and F. Pillichshammer, Bounds for the weighted $L^{p}$ discrepancy and tractability of integration, J. Complexity 19 (2003), no. 4, 529-547. MR1991981 (2004f:65030)

[Nie92] H. Niederreiter, Random number generation and quasi-Monte Carlo methods, CBMSNSF Regional Conference Series in Applied Mathematics, vol. 63, Society for Industrial and Applied Mathematics (SIAM), Philadelphia, PA, 1992. MR1172997 (93h:65008)

[Rag73] M. Raghavachari, Limiting distributions of Kolmogorov-Smirnov type statistics under the alternative, Ann. Statist. 1 (1973), 67-73. MR0346976 (49:11696)

[ST96] F. Schmid and M. Trede, An $L_{1}$-variant of the Cramér-von Mises test, Statist. Probab. Lett. 26 (1996), no. 1, 91-96. MR.1385667 (97a:62109)

[SW86] G.R. Shorack and J.A. Wellner, Empirical processes with applications to statistics, Wiley Series in Probability and Mathematical Statistics: Probability and Mathematical Statistics, John Wiley \& Sons, Inc., New York, 1986. MR0838963 (88e:60002)

[SW98] I.H. Sloan and H. Woźniakowski, When are quasi-Monte Carlo algorithms efficient for high-dimensional integrals?, J. Complexity 14 (1998), no. 1, 1-33. MR1617765 (99d:65384)

[SWW04] I.H. Sloan, X. Wang, and H. Woźniakowski, Finite-order weights imply tractability of multivariate integration, J. Complexity 20 (2004), no. 1, 46-74. MR2031558 (2004j:65034)

[vdV98] A.W. van der Vaart, Asymptotic statistics, Cambridge Series in Statistical and Probabilistic Mathematics, Cambridge University Press, Cambridge, 1998. MR.1652247 (2000c:62003) 
[vdVW96] A.W. van der Vaart and J.A. Wellner, Weak convergence and empirical processes, Springer Series in Statistics, Springer-Verlag, New York, 1996. MR1385671(97g:60035)

[vHKH97] A van Hameren, R. Kleiss, and J. Hoogland, Gaussian limits for discrepancies. I. Asymptotic results, Comput. Phys. Comm. 107 (1997), no. 1-3, 1-20. MR1488791 (99k:65009)

Dipartimento di Economia, Università degli Studi Dell'Insubria, Via Ravasi 2, 21100 VARESE, ItALY

E-mail address: cchoirat@eco.uninsubria.it

Dipartimento di Economia, Università degli Studi Dell'Insubria, Via Ravasi 2, 21100 VARESE, ITALY

E-mail address: rseri@eco.uninsubria.it 